\title{
Homotopy colimits - comparison lemmas for combinatorial applications
}

By Volkmar Welker ${ }^{1}$ ) and Günter M. Ziegler ${ }^{2}$ ) at Berlin, and Rade T. Živaljevic ${ }^{3}$ ) at Belgrade

\begin{abstract}
We provide a "toolkit" of basic lemmas for the comparison of homotopy types of homotopy colimits of diagrams of spaces over small categories. We show how this toolkit can be used in quite different fields of applications. We demonstrate this with respect to
\end{abstract}

1. Björner's "Generalized Homotopy Complementation Formula" [5],

2. the topology of toric varieties,

3. the study of homotopy types of arrangements of subspaces,

4. the analysis of homotopy types of subgroup complexes.

\section{Introduction}

The aim of this paper is to advertise homotopy colimit considerations for topological investigations in combinatorics. For this, we provide a toolkit and demonstrate its usefulness by a number of applications.

A diagram of spaces is a functor from a small category to the category of topological spaces. In various topological, geometric, algebraic and combinatorial situations one has to deal with structures that can profitably be interpreted as (co)limits or homotopy (co)limits of diagrams over small categories, specially over (finite) posets. In fact, if a space is written as a finite union of (simpler) pieces, then it is the colimit of a corresponding diagram of spaces. While (co)limits do not have good functorial properties in homotopy theory, they can usually be replaced by homotopy (co)limits (Puppe [32] may have been the first to exploit this). Homotopy (co)limits have much better functorial properties. Thus there is

\footnotetext{
1) Supported by Deutsche Forschungsgemeinschaft (DFG).

$\left.{ }^{2}\right)$ Supported by a "Gerhard-Hess-Förderpreis" of the DFG, which also provided support for the authors' joint work at the Konrad-Zuse-Zentrum für Informationstechnik Berlin (ZIB) in 1994.

$\left.{ }^{3}\right)$ Partially supported by the Serbian Science Foundation, Grant 04M03.
} 
a wide variety of techniques to manipulate diagrams of spaces in such a way that the homotopy (co)limit is preserved (up to homotopy type).

Basic work on homotopy (co)limits has been done by Segal [37], Bousfield and Kan [7], tom Dieck [42], Vogt [44], and Dwyer and Kan [11], [12]. See Hollender and Vogt [21] for a recent survey.

Two key results in this setting are the "Projection Lemma" [3], [7], XII.3.1(iv), [15], [37], [49] which sometimes allows one to replace colimits by homotopy colimits, and the "Homotopy Lemma" [42], [7], XII. 4.2, [44] which compares the homotopy types of diagrams over the same small category. These tools have found striking applications, for example, in the study of subspace arrangements [49], [41], [36].

The choice of contents for our "toolkit for the manipulation of homotopy colimits" is partially motivated by the usefulness of corresponding lemmas in the special case of order complexes (the discrete case, when the spaces of the diagram are points). In this case, there is a solid amount of theory available, which has proved to be extremely powerful and useful in quite diverse situations. The key result is the Quillen Fiber Theorem (Quillen's "Theorem A" [33], [34], see below). All other basic tools of the "homotopy theory of posets", such as the crosscut theorem, order homotopy theorem, complementation formulas, etc., can be derived from it. We refer to Björner [4] for an excellent account of the theory [4], Sect.10, for an extensive survey of applications [4], Part I, and for further references.

Homotopy limits have not found immediate applications in combinatorics and discrete geometry so far, and this is the reason why we restrict our attention to the case of homotopy colimits. Also note that many results about homotopy limits can be derived from the case of homotopy colimits by standard duality procedures (see Bousfield and Kan [7], XII. 4.1, and Hollender and Vogt [21], Sect.3).

We provide several applications of our methods to various areas within mathematics. As a first application, in the field of topological combinatorics, we present a new proof of a result by Björner on the homotopy type of complexes [5], which generalizes the Homotopy Complementation Formula of Björner and Walker [6], a tool which has proved to be very powerful in combinatorics. Since this proof affords the application of many of the techniques provided in this paper, we give a detailed exposition of it here.

Then we present a new view of toric varieties. Namely, we start with the observation that toric varieties are homeomorphic to homotopy colimits over the face poset of the fan defining the variety. This immediately leads to a spectral sequence to compute the homology of toric varieties isomorphic to one already employed by Danilov [9].

We derive a new "combinatorial formula" for the homotopy types of quite general arrangements (such as "Grassmannian" arrangements) that are associated to linear subspace arrangements by suitable functorial constructions. More briefly we cover two applications for which details are contained in other papers: We describe a new result on the homotopy type of the order complex of the poset $S_{p}(G)$ of non-trivial $p$-subgroups of a finite group $G$ [31], and we review results obtained by homotopy limit methods on the topology of subspace arrangements in [49], and provide the equivalence with the results of Vassiliev [43]. 


\section{Fundamental concepts and constructions}

2.1. Basic definitions and motivating examples of diagrams. In the following, all categories are small, so their objects and morphisms form sets. Any partially ordered set can be considered as a category "with morphisms pointing down", that is, for $x, y \in P$ there is a (unique) arrow $x \rightarrow y$ if and only if $x \geqq y$.

A diagram of spaces over a small category $A$ is a covariant functor $\mathscr{F}: A \rightarrow$ Top into the category Top of topological spaces. We denote for an object $a \in \operatorname{Obj}(A)$ the image under $\mathscr{F}$ by $F_{a}$ or by $F(a)$, and for a morphism $g: a \rightarrow b \in \operatorname{Mor}(A)$ the image $\mathscr{F}(g)$ by $f_{g: a \rightarrow b}$ or by $F(g)$. If there is a unique morphism $g: a \rightarrow b$ in $A$ between $a$ and $b$, then we write $f_{a b}$ for $f_{g: a \rightarrow b}$. A morphism $(F, \alpha): \mathscr{X} \rightarrow \mathscr{Y}$ of diagrams $\mathscr{X}: A \rightarrow T o p$ and $\mathscr{Y}: B \rightarrow T o p$ is a functor $F: A \rightarrow B$ together with a natural transformation $\alpha$ from $\mathscr{X}$ to $\mathscr{Y} \circ F=: F^{*}(\mathscr{Y})$. Given a diagram $\mathscr{F}$, the homotopy colimit hocolim $\mathscr{F}$ is a space associated to $\mathscr{F}$ by a homotopy mixing construction, see Section 2.2. Before we proceed with a reasonably detailed outline of the theory, we introduce several motivating examples of diagrams of spaces.

Constant diagram. For a topological space $X$ the constant diagram $\mathscr{X}_{A}$ is defined by sending each object of $A$ to the space $X$ and each morphism to the identity id : $X \rightarrow X$. Of particular interest is the case when $X=\{*\}$ is the one-point space. In this case the constant diagram $\mathscr{P} \mathscr{T}_{A}$ leads, via homotopy colimits, to the construction of the classifying space $B A$ of the category $A$. In the special case when $A=P$ is a partially ordered set - a poset for short - the classifying space $B P$ will be seen to coincide with the order complex $\Delta(P)$ of $P$.

Group diagram. Given a discrete group $G$, let $A_{G}$ be the category which consists of one single object and a morphism for each element $g \in G$. Then the classifying space of this category is the $K(G, 1)$-space $B A_{G} \cong B G$ of the group $G$. Its universal cover $E G$ is constructed in a similar way from the category whose objects are the elements of the group. Here for each pair of elements $g, h \in G$ the unique morphism from $g$ to $h$ is given by $h g^{-1}$. Finally, any $A_{G}$-diagram $\mathscr{X}: A_{G} \rightarrow T o p$ can be interpreted as a $G$-space $X$ and it turns out that hocolim ${ }_{A_{G}} \mathscr{X} \cong E G \times{ }_{G} X$.

Subspace diagrams. Let $U:=\left\{X_{i}\right\}_{i \in I}$ be a collection of subspaces of a topological space $X$. The intersection poset $P_{U}$ of the family $U$ is the partially ordered set of all nonempty intersections $\bigcap_{i \in J} X_{i}, J \subseteq I$, ordered by reversed inclusion. Regard $P_{U}$ as a small category. Then the subspace diagram associated to $U$ is the diagram $\mathscr{D}_{U}: P_{U} \rightarrow T o p$ sending each element of $P_{U}$ to the corresponding intersection $\bigcap_{i \in J} X_{i}$, with inclusions as morphisms. This class of examples can be used to see many results from topological combinatorics from a higher perspective (e.g. Borsuk's Nerve theorem [4]).

Arrangements as diagrams. An especially interesting class of examples arises if $X$ in the previous example is a linear space (affine space, sphere, projective space) and the family $U$ is an (affine, spherical, projective) subspace arrangement. The subspace diagrams that arise this way have been successfully used to deduce both new and old results about the homotopy and homology of these arrangements and their complements (see Ziegler and Živaljević [49], Schaper [36]). 
Orbit diagrams. Let $G$ be a Lie group acting on a space $X$ with finitely many orbit types. From this arises a natural diagram $\mathcal{O}: O_{G} \rightarrow T o p$ over the category $O_{G}$ of all $G$-orbits defined by $\mathcal{O}(G / H):=X^{H}$, see [14]. Recall that the orbit category $O_{G}$ is defined as the category with $\operatorname{Obj}\left(O_{G}\right):=\{G / H \mid H=\bar{H} \leqq G\}$ while the morphisms are $G$-equivariant maps $G / H \rightarrow G / Q$.

Generally any diagram over the orbit category $O_{G}$ will be referred to as an orbit diagram.

Toric diagrams. A toric diagram $\mathscr{D}$ is a diagram for which each space $D_{d}$ is the standard torus $T^{j}=S^{1} \times \cdots \times S^{1}, j \geqq 0$, and each morphism $d_{a b}: D_{a} \rightarrow D_{b}$ is a standard algebraic homomorphism, i.e., a homomorphism that arises from an integer matrix $K: \mathbb{R}^{i} \rightarrow \mathbb{R}^{j}, i=\operatorname{dim}\left(D_{a}\right), j=\operatorname{dim}\left(D_{b}\right)$. An important observation is that any compact (complex) toric variety can be interpreted as the homotopy colimit of a toric diagram over the face poset of a complete fan, see Section 5.3.

In order to avoid pathological behavior of the topological spaces involved and because this setting covers all application we can think of - in our combinatorial context - we assume that all spaces are compactly generated. For the same reason we restrict our attention to small categories which are not topologized. Note however that the general theory (see [21]) can be developed for the case of topological categories $A$ for which the $\operatorname{map} \operatorname{Obj}(A) \hookrightarrow \operatorname{Mor}(A)$ is a cofibration.

2.2. Limit space constructions. We will now discuss several constructions of "limit spaces" from a diagram $\mathscr{D}$. For that we recall the notion of a simplicial space and its geometric realization. The references provide more information about simplicial sets and spaces, their geometric realizations, and other general categorical constructions used in this paper. Nevertheless, we try to be as self-contained as possible.

The category $\operatorname{Ord}$ has as objects the finite ordinals $[n]:=\{0, \ldots, n\}, n \geqq 0$; its morphisms are the non-decreasing maps $f:[n] \rightarrow[m]$ (i.e., $f(i) \leqq f(i+1)$ ). The set of morphisms of $\operatorname{Ord}$ is generated [25], p.4, [19], II. 2.2 by the maps $\delta_{n}^{i}$ and $\sigma_{n}^{i}$, where $\delta_{n}^{i}:[n-1] \rightarrow[n]$ is the increasing injection that does not assume the value $i$ (for $0 \leqq i \leqq n$ ), and $\sigma_{n}^{i}:[n+1] \rightarrow[n]$ is the non-decreasing surjection that assumes the value $i$ twice (for $0 \leqq i \leqq n)$.

A simplicial space is a contravariant functor F:Ord $\rightarrow$ Top. A simplicial set is a contravariant functor F : Ord $\rightarrow$ Set. We will not distinguish between simplicial sets and simplicial spaces equipped with the discrete topology. SimplicialSets and SimplicialSpaces are categories with the "obvious" morphisms, that is, whose morphisms are the natural transformations between functors $\mathrm{Ord} \rightarrow$ Set resp. Ord $\rightarrow$ Top .

There is a simple geometric realization functor $\mathrm{R}: \operatorname{Ord} \rightarrow$ Top that associates with $[n]$ the standard $n$-dimensional simplex

$$
\Delta_{n}:=\left\{\left(t_{0}, t_{1}, \ldots, t_{n}\right) \in \mathbb{R}^{n+1}: 0 \leqq t_{0}, t_{1}, \ldots, t_{n} \leqq 1, \sum_{i=0}^{n} t_{i}=1\right\}
$$


and associates with $\delta_{n}^{i}$ and $\sigma_{n}^{i}$ the "face operators" $\mathrm{R}\left(\delta_{n}^{i}\right)$ resp. the "degeneracy operators" $\mathrm{R}\left(\sigma_{n}^{i}\right)$. (Viewing $\operatorname{Ord}$ as the category of finite chains and order preserving maps, the functor $\mathrm{R}$ associates with chains their order complexes and with order preserving maps the corresponding simplicial maps.)

Now we are able to review some important "limit space" or "total space" constructions arising from categories and diagrams.

Geometric realization. Given a simplicial space $\mathrm{F}$ one defines its geometric realization $|\mathrm{F}|$. Denote by $\mathrm{F}_{n}:=\mathrm{F}([n])$ the image of $[n]$ under $\mathrm{F}$, by $d_{n}^{i}$ the image of $\delta_{n}^{i}$ under $\mathrm{F}$ and by $s_{n}^{i}$ the image of $\sigma_{n}^{i}$ under $\mathrm{F}$. Then $|\mathrm{F}|$ is obtained as the quotient of the disjoint union $\underset{n \geq 0}{\uplus} F_{n} \times \Delta_{n}$ modulo the equivalence relation " " defined by

$$
\begin{array}{ll}
\left(d_{n}^{i}(x), p\right) \sim\left(x, \mathrm{R}\left(\delta_{n}^{i}\right)(p)\right) & \text { for } x \in \mathrm{F}_{n} \text { and } p \in \Delta_{n-1}, \\
\left(s_{n}^{i}(x), p\right) \sim\left(x, \mathrm{R}\left(\sigma_{n}^{i}\right)(p)\right) & \text { for } x \in \mathrm{F}_{n} \text { and } p \in \Delta_{n+1} .
\end{array}
$$

With this geometric realization, $|\cdot|$ is a functor from SimplicialSpaces to Top.

Classifying spaces. If $A$ is any small category, then there is a natural simplicial set $\mathrm{F}_{A}:$ Ord $\rightarrow$ Set associated to it. Namely, the image of $[n]$ under $\mathrm{F}_{A}$ consists of all sequences $\alpha_{n} \stackrel{f_{n}}{\cdots} \cdots f_{1} \alpha_{0}$ of objects $\alpha_{i} \in \operatorname{Obj}(A)$ and maps $f_{i} \in \operatorname{Mor}_{A}\left(\alpha_{i-1}, \alpha_{i}\right)$ in the category $A$. The maps $d_{n}^{i}$ are given by

$$
d_{n}^{i}\left(\alpha_{n} \stackrel{f_{n}}{\longleftarrow} \cdots \stackrel{f_{1}}{\longleftarrow} \alpha_{0}\right)= \begin{cases}\alpha_{n} \stackrel{f_{n}}{\longleftarrow} \cdots \alpha_{i} \stackrel{f_{i}}{\longleftarrow} \alpha_{i-1} \cdots \stackrel{f_{2}}{\longleftarrow} \alpha_{1}, & i=0, \\ \alpha_{n} \stackrel{f_{n}}{\longleftarrow} \cdots \alpha_{i+1} \stackrel{f_{i+1}^{\circ} f_{i}}{\longleftarrow} \alpha_{i-1} \cdots f_{1} & \alpha_{0}, \quad 1 \leqq i \leqq n-1, \\ \alpha_{n-1} \stackrel{f_{n-1}}{\leftrightarrows} \cdots \alpha_{i} \stackrel{f_{i}}{\longleftarrow} \alpha_{i-1} \cdots \stackrel{f_{1}}{\longleftarrow} \alpha_{0}, & i=n .\end{cases}
$$

The maps $s_{n}^{i}$ are given by

$$
s_{n}^{i}\left(\alpha_{n} \stackrel{f_{n}}{\longleftarrow} \cdots \stackrel{f_{1}}{\longleftarrow} \alpha_{0}\right)=\alpha_{n} \stackrel{f_{n}}{f^{\prime}} \cdots \stackrel{f_{j+1}}{\longleftarrow} \alpha_{i} \stackrel{\mathrm{id}_{\alpha_{i}}}{\longleftarrow} \alpha_{i} \stackrel{f_{i}}{\longleftarrow} \cdots f_{1} \alpha_{0} .
$$

The geometric realization of the simplicial set $\mathrm{F}_{A}$ is the classifying space $B(A)$ of the category $A$.

Colimit. The colimit of a diagram $\mathscr{D}$ is the topological space obtained from the direct sum $\underset{a \in A}{\bigoplus} D_{a}$ modulo the equivalence relation " $\sim$ " generated by $x \sim y$ for $x \in D_{a}$, $y \in D_{b}$ if $d_{g: a \rightarrow b} \underset{a \in A}{ }(x)=y$ for some $g: a \rightarrow b$. We write colim $\mathscr{D}$ for the colimit of the diagram $\mathscr{D}$.

Homotopy colimit. The homotopy colimit of a diagram $\mathscr{D}$ is defined as the quotient of the direct sum $\underset{a \in A}{\uplus} B\left(A_{\downarrow a}\right) \times D_{a}$ by an equivalence relation " $\sim$." Here $A_{\downarrow a}$ denotes, for a given category $A$ and $a \in \operatorname{Obj}(A)$, the category of all arrows $a \rightarrow b$ emanating from $a$ with commutative triangles as morphisms between objects $a \rightarrow b_{1}$ and $a \rightarrow b_{2}$. If $A=P$ is 
a partially ordered set, then $A_{\downarrow a}=P_{\leqq a}=\{p \in P \mid p \leqq a\}$. The equivalence relation " $\sim$ " is defined as follows. For all morphisms $f: a \rightarrow b$ consider the maps:

$$
\begin{aligned}
& \alpha:\left\{\begin{array}{ccc}
B\left(A_{\downarrow b}\right) \times D_{a} & \rightarrow & B\left(A_{\downarrow b}\right) \times D_{b}, \\
(p, x) & \mapsto & \left(p, d_{f: a \rightarrow b}(x)\right),
\end{array}\right. \\
& \beta:\left\{\begin{array}{ccc}
B\left(A_{\downarrow b}\right) \times D_{a} & \rightarrow & B\left(A_{\downarrow a}\right) \times D_{a}, \\
(p, x) & \mapsto & (B(f)(p), x) .
\end{array}\right.
\end{aligned}
$$

Then " $\sim$ " is the transitive closure of $\alpha(p, x) \sim \beta(p, x)$.

If it is important to emphasize the indexing category $A$, then the homotopy colimit of a diagram $\mathscr{D}$ is denoted by hocolim $A$.

Bar construction. A very general construction of a limit space is a construction given by Hollender and Vogt [21]. Given diagrams $\mathscr{D}: A^{\text {op }} \rightarrow$ Top and $\mathscr{E}: A \rightarrow$ Top over a category $A$ and its opposite category $A^{\text {op }}$, there is an associated total space $B(\mathscr{D}, A, \mathscr{E})$. Its construction generalizes all limit space constructions presented so far (except for the colimit construction). To the data $\mathscr{D}$ and $\mathscr{E}$ one associates a simplicial set $\Delta(\mathscr{D}, A, \mathscr{E})$. The space of $n$-simplices of $\Delta(\mathscr{D}, A, \mathscr{E})$ is the space of all "threads"

$$
\left(x, \alpha_{n} \stackrel{f_{n}}{\longleftarrow} \alpha_{n-1} \stackrel{f_{n-1}}{\longleftarrow} \cdots f_{1} \alpha_{0}, y\right),
$$

where $x \in D_{\alpha_{n}}$ and $y \in E_{\alpha_{0}}$. More precisely, this space can be described as the space $\uplus_{\alpha_{n}} D_{\alpha_{n}} \times E_{\alpha_{0}}$, where the direct sum is taken over all $n$-chains $\alpha_{n} \longleftarrow f_{n} \cdots \longleftarrow f_{1} \alpha_{0}$ of morphisms in $A$. The maps $d_{n}^{i}(0 \leqq i \leqq n)$ are given by

$$
\begin{aligned}
& d_{n}^{i}\left(x, \alpha_{n} \stackrel{f_{n}}{\longleftarrow} \cdots \stackrel{f_{1}}{\longleftarrow} \alpha_{0}, y\right) \\
& = \begin{cases}\left(x, \alpha_{n} \stackrel{f_{n}}{\leftrightarrows} \cdots \alpha_{i} \stackrel{f_{i}}{\longleftarrow} \alpha_{i-1} \cdots \stackrel{f_{2}}{\longleftarrow} \alpha_{1}, e_{f_{1}}(y)\right), & i=0, \\
\left(x, \alpha_{n} \stackrel{f_{n}}{\cdots} \cdots \alpha_{i+1} \stackrel{f_{i+1} f_{i}}{\longleftarrow} \alpha_{i-1} \cdots \stackrel{f_{1}}{\longleftarrow} \alpha_{0}, y\right), & 1 \leqq i \leqq n-1, \\
\left(d_{f_{n}}(x), \alpha_{n-1} \stackrel{f_{n-1}}{\longleftarrow} \cdots \alpha_{i} \stackrel{f_{i}}{\longleftarrow} \alpha_{i-1} \cdots \stackrel{f_{1}}{\longleftarrow} \alpha_{0}, y\right), & i=n .\end{cases}
\end{aligned}
$$

The maps $s_{n}^{i}(0 \leqq i \leqq n)$ are given by

$$
s_{n}^{i}\left(x, \alpha_{n} \stackrel{f_{n}}{\longleftarrow} \cdots \stackrel{f_{1}}{\longleftarrow} \alpha_{0}, y\right)=\left(x, \alpha_{n} \stackrel{f_{n}}{\longleftarrow} \cdots \stackrel{f_{i+1}}{\longleftarrow} \alpha_{i} \stackrel{\mathrm{id}_{\alpha_{i}}}{\longleftarrow} \alpha_{i} \cdots \stackrel{f_{1}}{\longleftarrow} \alpha_{0}, y\right) .
$$

Denote by $B(\mathscr{D}, A, \mathscr{E})$ the geometric realization of the simplicial space $\Delta(\mathscr{D}, A, \mathscr{E})$. The homotopy colimit and the classifying space are special cases of the geometric realization of simplicial spaces of the form $\Delta(\mathscr{D}, A, \mathscr{E})$. Namely, let $\mathscr{P} \mathscr{T}_{A}$ be the diagram over the category $A$ that assigns to each object $a$ in $A$ the one-point space $\{*\}$. Then the classifying space of the category $A$ is the geometric realization of $\Delta\left(\mathscr{P} \mathscr{T}_{A^{\mathrm{op}}}, A, \mathscr{P} \mathscr{T}\right)$. For a diagram $\mathscr{D}: A \rightarrow$ Top the homotopy colimit hocolim $\mathscr{D}$ is homeomorphic to the space $B\left(\mathscr{P} \mathscr{T}_{A^{\text {op }}}, A, \mathscr{D}\right)$ (see [21]). 
We may observe that for every diagram $\mathscr{D}$ over a category that is a poset $P$ there is a canonical partial order on $\mathbf{P}:=\bigoplus_{p \in P} D_{p}$ defined by setting $x \geqq x^{\prime}$ for $x \in D_{p}$ and $x^{\prime} \in D_{p^{\prime}}$ if and only if $p \geqq p^{\prime}$ and $d_{p p^{\prime}}(x)=x^{\prime}$. With this definition there is a canonical bijection $\Delta(\mathbf{P}) \leftrightarrow \operatorname{hocolim}(\mathscr{D})$; however, the topology on the two spaces is different: in fact, with the usual topology on simplicial complexes the subspaces $D_{p} \subseteq \Delta(\mathbf{P})$ get a discrete topology. Note however, see Section 4.1, that there exists a convenient geometric model for hocolim $(\mathscr{D})$ realizing this space as a subspace of the join $J(\mathscr{D}):=\underset{p \in P}{*} D_{p}$. This construction can be seen as a natural extension of the order complex construction to diagrams over posets.

Examples 2.1. Here are some simple examples for the construction of homotopy colimits of diagrams over posets.

(i) If $D_{p}$ is a one-point space for all $p \in P$, i.e. $\mathscr{D}=\mathscr{P} \mathscr{T}_{P}$, then hocolim $\mathscr{D}$ is (isomorphic to) the order complex of $P$.

(ii) If $P=\{p\}$ is a one-element poset, then hocolim $\mathscr{D}=D_{p}$.

(iii) If $P=\left\{p, p^{\prime}\right\}$ has two points and $p^{\prime}>p$, then hocolim $\mathscr{D}$ is the mapping cylinder of the map $f=d_{p^{\prime} p}: D_{p^{\prime}} \rightarrow D_{p}$.

(The mapping cylinder is homotopy equivalent to the image space $D_{p}$, which is the colimit of the diagram.)

(iv) If $P=\left\{p, p^{\prime}\right\}$ and $p, p^{\prime}$ are not comparable, then hocolim $\mathscr{D}=D_{p} \uplus D_{p^{\prime}}$ is the disjoint union.

(In this case it agrees with the colimit colim $\mathscr{D}$ of the diagram.)

(v) Let $P=\left\{p, p^{\prime}, p^{\prime \prime}\right\}$ be the three-element poset with $p \geqq p^{\prime}, p \geqq p^{\prime \prime}$, for which $p^{\prime}$ and $p^{\prime \prime}$ are incomparable, and let $\mathscr{D}$ be a diagram over $P$ with $D_{p^{\prime \prime}}=\{*\}$ a one-point space. Then the homotopy colimit hocolim $(\mathscr{D})$ is homeomorphic to the mapping cone of the map $d_{p p^{\prime}}: D_{p} \rightarrow D_{p^{\prime}}$.

(vi) If $\mathscr{D}$ is a diagram such that the spaces $D_{p}$ are identical, and the maps are identity maps, then hocolim $\mathscr{D} \cong \Delta(P) \times D_{p}$.

(The colimit of such a diagram is $D_{p}$, if $P$ is connected.)

\subsection{Simplicial homotopy lemma and the gluing lemma.}

Definition 2.2. A simplicial space F : Ord $\rightarrow$ Top is called good if the inclusion map $\sigma(F[n]) \rightarrow F([n+1])$ is a cofibration where $\sigma(F([n])):=\bigcup_{i=0}^{n} \operatorname{Im}\left(F\left(\sigma_{n}^{i}\right)\right)$ is the space of all degenerated simplices in $F([n+1])$. According to Lillig's union theorem for cofibrations [23], $\mathrm{F}$ is good if for any surjective map $\sigma:[n+1] \rightarrow[n]$ in Ord the inclusion $\operatorname{Im}(F(\sigma)) \rightarrow F([n+1])$ is a closed cofibration. 
Proposition 2.3 (Simplicial Homotopy Lemma). Let A, B be two good simplicial spaces. Let $\mathrm{F}: \mathrm{A} \rightarrow \mathrm{B}$ be a map of simplicial spaces. If $\mathrm{F}_{n}: \mathrm{A}_{n} \rightarrow \mathrm{B}_{n}$ is a homotopy equivalence for all $n$, then the induced map

$$
|\mathrm{F}|:|\mathrm{A}| \rightarrow|\mathrm{B}|
$$

is a homotopy equivalence.

Good simplicial spaces were defined by Segal [38], Appendix. In [27] and in [26] the closely related, albeit not coinciding, classes of proper and strictly proper spaces are introduced. Proofs of Proposition 2.3 can be found in either of the references mentioned above.

The Gluing Lemma is a key result. It has repeatedly been used in inductive proofs of results establishing that two spaces are homotopy equivalent; a notable example is the proof of Proposition 2.3. This lemma is a basic example for an important general principle which informally says that a local homotopy equivalence is also a global homotopy equivalence - see the Homotopy Lemma 3.7.

Lemma 2.4 (Gluing Lemma [8], [42]). Consider the commutative diagram of spaces

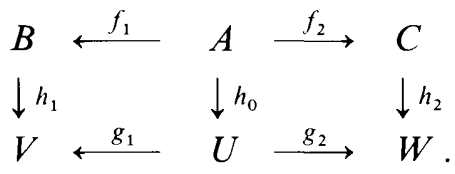

Assume that $h_{0}, h_{1}, h_{2}$ are homotopy equivalences and that either both $f_{1}$ and $g_{1}$ are cofibrations or both $f_{2}$ and $g_{2}$ are cofibrations. Let $X=B \cup_{A} C$ (resp., $\left.Y=V \cup_{U} W\right)$ denote the colimit (or push-out) of the diagram formed by the maps $\left(f_{1}, f_{2}\right)$ (resp., $\left.\left(g_{1}, g_{2}\right)\right)$. Then the induced map $h: X \rightarrow Y$ is also a homotopy equivalence.

\section{Comparison results for diagrams of spaces}

In this section we set up the toolkit for applications of diagrams of spaces. In the subsequent section we will show how these very general results specialize to forms applicable in various combinatorial situations where the emphasis is on diagrams over (locally) finite posets. The toolkit consists of a sequence of propositions and lemmas most of which are part of standard tools of an algebraic topologist. These results allows us to recognize a topological space as the homotopy colimit of a diagram (Projection Lemma), to check if a given morphism $(F, \alpha): \mathscr{X} \rightarrow \mathscr{Y}$ of diagrams induces a homotopy equivalence

$$
\hat{\alpha}: \operatorname{hocolim}_{A} \mathscr{X} \rightarrow \operatorname{hocolim}_{B} \mathscr{Y}
$$

of the corresponding homotopy colimits (Homotopy Lemma), to change the underlying small category of the diagram without changing the homotopy type of the homotopy colimit, to "compute" a standard, combinatorial form of the space hocolim ${ }_{A} \mathscr{X}$ (Wedge Lemma), etc. 


\subsection{Projection, wedge and the quasifibration lemma.}

Proposition 3.1 (Projection Lemma [3], [49]). Let $P$ be a finite poset and let $\mathscr{X}: P \rightarrow$ Top a diagram having the property that for each $p \in P$ the induced map $\operatorname{colim}_{q>p} X(q) \rightarrow X(p)$ is a closed cofibration. Then the natural map

$$
\operatorname{hocolim}_{P} \mathscr{X} \rightarrow \operatorname{colim}_{P} \mathscr{X}
$$

is a homotopy equivalence.

Remark. A more general form of the Projection Lemma has been established for the so called free diagrams over arbitrary small categories, see [14] and [15], Appendix HL. The first "Projection Lemma" was established by Segal [37] for numerable coverings of topological spaces.

The next result that we are heading for, the Wedge Lemma, has been formulated and used before in the case of diagrams over posets [49]. In order to formulate and prove a more general form of this lemma we need two preliminary definitions.

Definition 3.2. A diagram $\mathscr{X}: A \rightarrow$ Top is called a diagram with constant maps if for all $a, b \in \operatorname{Obj}(A)$ and any nonidentity morphism $f: a \rightarrow b, f \neq \mathrm{id}_{a}$, the map $X(f): X(a) \rightarrow X(b)$ is a constant map. A diagram with coherent constant maps is a diagram with constant maps with the additional property that for any object $a \in \operatorname{Obj}(A)$ there exists a point $x_{a} \in X(a)$ such that for any morphism $f: b \rightarrow a, f \neq \mathrm{id}_{a}$, the one element set $\operatorname{Im}(X(f))$ is precisely $\left\{x_{a}\right\}$. In addition it is assumed that all spaces $X(a)$ are well pointed [8], which means that $\left(X(a),\left\{x_{a}\right\}\right)$ is a cofibration pair. If the category $A$ has an initial object $a$, (i.e., an object such that for each $b \in \operatorname{Obj}(A)$ there is a unique morphism from $a$ to $b$ ) then a diagram with constant maps over $A$ is called an initial diagram with constant maps.

Note that an initial diagram with constant maps is automatically a diagram with coherent constant maps.

Recall, see Section 2.2, that for a given category $A$ and $a \in \operatorname{Obj}(A)$, the undercategory $A_{\downarrow a}$, often denoted by $a \backslash A$ or $a \downarrow \mathrm{Id}$, is the category with objects

$$
\operatorname{Obj}\left(A_{\downarrow a}\right):=\underset{b \in \operatorname{Obj}(A)}{\bigoplus} \operatorname{Mor}(a, b)
$$

and an arrow from $a \stackrel{f}{\longrightarrow} x$ to $a \stackrel{g}{\longrightarrow} y$ for each commutative triangle formed by $f, g$ and a morphism $x \stackrel{h}{\longrightarrow} y, g=h \circ f$.

Definition 3.3. Given $c \in \operatorname{Obj}(A)$, the truncated category obtained by deleting $c$ and all incident morphisms is denoted by $A \backslash c$. If we delete from $A_{\downarrow a}$ the initial object $a \stackrel{\text { id }}{\longrightarrow} a$ and all incident morphisms we obtain the truncated undercategory $A_{\downarrow} \backslash \backslash \mathrm{id}_{a}$ which is denoted by $A_{\Downarrow a}$. and $P_{<a}$.

Specially, if $A$ is a poset category $P$, then $A_{\downarrow a}$ and $A_{\Downarrow a}$ are the poset categories $P_{\leqq a}$

9 Journal für Mathematik. Band 509 
Lemma 3.4. Let $\mathscr{X}$ be an initial diagram with constant maps over a category $A$ with $a$ as an initial object. If $X(b)$ is a one-element space for each object $b \neq a$, then the homotopy colimit of this diagram has the following join decomposition:

$$
\operatorname{hocolim}_{A} \mathscr{X} \simeq X(a) * B(A \backslash \backslash a),
$$

where $B(A \backslash a)$ is the classifying space of $A \backslash a$. Moreover, if $X(a)$ is not a singleton then these spaces are homeomorphic.

Proof. For an object $b \in \operatorname{Obj}(A)$ let $f_{b} \in \operatorname{Mor}(a, b)$ be the unique morphism connecting $a$ and $b$ (e.g., $f_{a}=\mathrm{id}_{a}$ ). If $g \in \operatorname{Mor}(b, a)$ then $g \circ f_{b}=\mathrm{id}_{a}$ hence $X(g) \circ X\left(f_{b}\right)=\mathrm{id}_{X(a)}$. If $b \neq a$ this is possible only if $X(a)$ consists of a single element. In this case hocolim $_{A} \mathscr{X}=B(A)$ and it is well known [37] (see also Section 3.3) that $B(A)$ is contractible if $A$ has an initial object. On the other hand the right hand side is obviously contractible.

If $|X(a)|>1$ then the only non-identity morphisms with $a$ as the domain or codomain are of the form $f_{b} \in \operatorname{Mor}(a, b)$ for some $b \in \operatorname{Obj}(A), b \neq a$. In this case the formula hocolim $_{A} \mathscr{X} \cong X(a) * B(A \backslash a)$ follows by the definition of the homotopy colimit through the bar-construction.

Remark. There are interesting examples of diagrams $\mathscr{X}: A \rightarrow$ Top, see [49] and Section 5.4, which have the property that for all $a, b \in \operatorname{Obj}(A)$ and $f: a \rightarrow b, f \neq \mathrm{id}_{a}$, the map $X(f): X(a) \rightarrow X(b)$ is homotopically trivial. Typically these diagrams can be transformed, usually with the aid of the Homotopy Lemma (Proposition 3.7), into diagrams with coherent constant maps without affecting the homotopy type of the homotopy colimit. The following lemma shows that in these cases we can actually "compute" these homotopy types.

Proposition 3.5 (Wedge Lemma). Suppose that $\mathscr{X}$ is a diagram with coherent constant maps over A. If the category $A$ is connected then the homotopy colimit of $\mathscr{X}$ has the wedge decomposition

$$
\operatorname{hocolim}_{A} \mathscr{X} \cong B(A) \vee \bigvee_{a \in \mathbf{O b j}(A)} X(a) * B\left(A_{\Downarrow a}\right)
$$

(It is assumed that the base point of $X(a) * B\left(A_{\Downarrow a}\right)$ is the base point of its subspace $X(a)$ while the base point of the connected $C W$-complex $B(A)$ is arbitrary.)

Proof. For each $a \in \operatorname{Obj}(A)$ we consider two diagrams:

- The initial diagram with constant maps $X[a]: A_{\downarrow a} \rightarrow T o p$, and $f_{a \rightarrow b} \mapsto\left\{x_{b}\right\}$ if $f_{a \rightarrow b} \neq \mathrm{id}_{a}$ and $\mathrm{id}_{a} \mapsto X(a)$. The maps are the obvious constant maps.

- The diagram with coherent constant maps $Y[a]: A \rightarrow T o p$, and $b \mapsto\left\{x_{b}\right\}$ if $b \neq a$ and $a \mapsto X(a)$. Again the maps are the obvious constant maps.

Since $\mathscr{X}$ is a diagram with coherent constant maps, there exist maps of diagrams $e_{a}: Y[a] \rightarrow \mathscr{X}$ and $p_{a}: \mathscr{X} \rightarrow Y[a]$ such that $p_{a} \circ e_{a}=\mathrm{id}_{Y[a]}$. This implies that $\mathscr{H}_{a}:=\operatorname{hocolim}_{A} Y[a]$ is a retract of $\mathscr{H}:=\operatorname{hocolim}_{A} \mathscr{X}$. In particular, $\mathscr{H}_{a}$ is a closed subspace of $\mathscr{H}$. Obviously, $\mathscr{H}=\bigcup_{a \in \mathrm{Obj}(A)} \mathscr{H}_{a}$. Moreover, $\mathscr{H}$ is the colimit of the covering by the $\mathscr{H}_{a}$. 
In other words a subset $F$ of $\mathscr{H}$ is closed if and only if $\mathscr{H}_{a} \cap F$ is closed in $\mathscr{H}_{a}$ for all $a \in \operatorname{Obj}(A)$. This follows from the fact that this is true on the level of simplicial spaces and that the geometric realization functor commutes with colimits.

Each space $\mathscr{H}_{a}$ is homeomorphic to the push-out of the diagram:

$$
\operatorname{hocolim}_{A_{\downarrow} a} X[a] \longleftarrow B\left(A_{\downarrow a}\right) \longrightarrow B(A) .
$$

From here we deduce that $\mathscr{H}=\operatorname{hocolim}_{A} \mathscr{X}$ is homeomorphic to the push-out of the diagram

$$
\underset{a \in \operatorname{Obj}(A)}{\uplus} \operatorname{hocolim}_{A_{\downarrow}} X[a] \longleftarrow \bigoplus_{a \in \operatorname{Obj}(A)} B\left(A_{\downarrow a}\right) \longrightarrow B(A) .
$$

Each of the spaces $B\left(A_{\downarrow a}\right)$ is contractible to its base point $\mathrm{id}_{a}$. We conclude from the Gluing Lemma 2.4 that the push-out of (2) is homotopy equivalent to the push-out of the following diagram:

$$
\bigoplus_{a \in \mathrm{Obj}(A)} \operatorname{hocolim}_{A_{\lfloor a}} X[a] \longleftarrow \biguplus_{a \in \mathrm{Obj}(A)}\left\{\operatorname{id}_{a}\right\} \stackrel{a}{\longrightarrow} B(A)
$$

By assumption $B(A)$ is connected which implies that $\alpha$ is homotopic to a constant map. This observation together with the Gluing Lemma 2.4 and 3.4 complete the proof.

Note that if the category $A$ is not connected, then the formula from Proposition 3.5 applies to the diagrams induced on each of the connected components.

The obvious "collapsing" map of diagrams $\alpha: \mathscr{X} \rightarrow \mathscr{P} \mathscr{T}_{A}$ where $\mathscr{X}$ is an arbitrary $A$-diagram and $\mathscr{P} \mathscr{T}_{A}$ is the constant point-diagram over $A$, induces a map $\hat{\alpha}$ : hocolim ${ }_{A} \mathscr{X} \rightarrow B(A)$. Under certain strong conditions this map turns out to be a quasifibration, a concept introduced by Dold and Thom [10]. A map $f: X \rightarrow Y$ between topological spaces is a quasifibration if for any $y \in Y$ and $x \in f^{-1}(y)$ the induced maps $f_{*}: \pi_{i}\left(X, f^{-1}(y), x\right) \rightarrow \pi_{i}(Y, y)$ are isomorphisms for all $i \geqq 0$. The following proposition is essentially due to Quillen [33].

Proposition 3.6 (Quasifibration Lemma [33], §1). If $\mathscr{X}$ is an A-diagram such that all the maps $X(f): X(a) \rightarrow X(b), f \in \operatorname{Mor}_{A}(a, b)$ are homotopy equivalences, then the natural projection map hocolim ${ }_{A} \mathscr{X} \rightarrow B(A)$ is a quasifibration. In particular, if $B(A)$ is contractible, then for every $a \in \operatorname{Obj}(A)$ the map $X(a) \rightarrow$ hocolim $_{A} \mathscr{X}$ is a homotopy equivalence.

3.2. From local to global homotopy equivalences. This is the first circle of results about homotopies between homotopy colimits of diagrams.

Proposition 3.7 (Homotopy Lemma). Suppose that $\mathscr{X}$ and $\mathscr{Y}$ are diagrams over $C$ and $\alpha=(\mathrm{id}, \alpha): \mathscr{X} \rightarrow \mathscr{Y}$ is a morphism such that $\alpha(c): X(c) \rightarrow Y(c)$ is a (weak) homotopy equivalence for all $c \in \operatorname{Obj}(C)$. Then the induced map

$$
\hat{\alpha}: \operatorname{hocolim}_{C} \mathscr{X} \rightarrow \operatorname{hocolim}_{C} \mathscr{Y}
$$

is also a (weak) homotopy equivalence. 
This Homotopy Lemma is a central result about diagrams of spaces. As noted above it has evolved from the Gluing Lemma, Proposition 2.4, in the papers of tom Dieck [42], Bousfield and Kan [7], XII. 4.2, Vogt [44] and others. Both the Homotopy Lemma and the following more general result from [21] for the bar construction (as discussed in Section 2.2) can be deduced from the results stated in Section 2.

Proposition 3.8. Let $\mathscr{X}, \mathscr{X}^{\prime}: C \rightarrow$ Top and $\mathscr{Y}, \mathscr{Y}^{\prime}: C^{\mathrm{op}} \rightarrow$ Top be diagrams. Suppose $(\mathrm{id}, \alpha): \mathscr{X} \rightarrow \mathscr{X}^{\prime}$ and $(\mathrm{id}, \beta): \mathscr{Y} \rightarrow \mathscr{Y}^{\prime}$, are morphisms of diagrams such that $\alpha(c): X(c) \rightarrow X^{\prime}(c)$ and $\beta(c): Y(c) \rightarrow Y^{\prime}(c)$ are homotopy equivalences for all $c \in \operatorname{Obj}(C)$. Then the induced map

$$
B(\beta, \text { id }, \alpha): B(\mathscr{Y}, C, \mathscr{X}) \rightarrow B\left(\mathscr{Y}^{\prime}, C, \mathscr{X}^{\prime}\right)
$$

is also a homotopy equivalence.

The Homotopy Lemma is a very convenient tool if we want to compare homotopy types of homotopy colimits of two diagrams over the same category $C$. For comparison of homotopy colimits of diagrams over different categories we have at our disposal the following two elegant results. They both describe possible changes of the indexing category of a diagram that do not affect the homotopy type of the homotopy colimit.

Definition 3.9. Given a functor $F: C \rightarrow D$, the pullback functor $F^{*}$ associates a $C$ diagram with every $D$-diagram: It is defined by $F^{*}(\mathscr{Y}):=\mathscr{Y} \circ F$ for any $D$-diagram $\mathscr{Y}$. If $d \in \operatorname{Obj}(D)$ let $d \downarrow F$ be the category which has as objects all arrows of the form $d \rightarrow F(c)$ for some $c \in \operatorname{Obj}(C)$ and for morphisms between arrows $d \stackrel{f}{\longrightarrow} F(c)$ and $d \stackrel{f}{\longrightarrow} F\left(c^{\prime}\right)$ commutative triangles $F(h) \circ f=g$ where $h \in \operatorname{Mor}_{C}\left(c, c^{\prime}\right)$.

If $F=\mathrm{Id}$ is the identity functor we recover in $d \downarrow$ Id the definition of undercategory from Definition 3.3. Dually to Definition 3.9 one defines the category $d \uparrow F$. Again in the case $F=$ Id we obtain the "overcategory" $D_{\uparrow d}$.

Proposition 3.10 (Cofinality Theorem [7], XI. 9.2, [12], [21]). If a functor $F: C \rightarrow D$ is right cofinal, i.e., if the classifying space $B(d \downarrow F)$ is homotopically trivial for all $d \in D$, then the induced map

$$
\operatorname{hocolim}_{C} F^{*} \mathscr{Y} \rightarrow \operatorname{hocolim}_{D} \mathscr{Y}
$$

is a homotopy equivalence.

The (Segal's) homotopy pushdown functor $F_{h_{*}}$ associates a $D$-diagram to any $C$-diagram $\mathscr{X}: C \rightarrow T o p$, [38], [21].

Definition 3.11. For every category $D$ there is a tautological $\left(D^{\mathrm{op}} \times D\right)$-diagram $\mathscr{D}_{(\cdot, \cdot)}: D^{\mathrm{op}} \times D \rightarrow$ Top of discrete spaces defined by $\left(d_{1}, d_{2}\right) \mapsto \operatorname{Mor}_{D}\left(d_{1}, d_{2}\right)$. If $d \in \operatorname{Obj}(D)$ 
is fixed, then let $\mathscr{D}_{(d, \cdot)}: D \rightarrow T o p$ be the $D$-diagram defined by $x \mapsto \operatorname{Mor}_{D}(d, x)$. Similarly, for a fixed $d^{\prime} \in \operatorname{Obj}(D)$, the $D^{\text {op }}$-diagram defined by $x \mapsto \operatorname{Mor}_{D}\left(x, d^{\prime}\right)$ is denoted by $\mathscr{D}_{\left(,, d^{\prime}\right)}$. Given a functor $F: C \rightarrow D$ and $d \in \operatorname{Obj}(D)$, the $D^{\text {op }}$-diagram $\mathscr{D}_{(\cdot, d)}$ is, via $F$, seen also as a $C^{\text {op }}$-diagram, so the space $B\left(\mathscr{D}_{(\cdot, d)}, C, \mathscr{X}\right)$ is well defined. The assignment $d \mapsto B\left(\mathscr{D}_{(\cdot, d)}, C, \mathscr{X}\right)$ defines a $D$-diagram $F_{h_{*}}(\mathscr{X})$ which is called the homotopy pushdown of $X$ or the left homotopy Kan extension along $F$.

Proposition 3.12 (Homotopy Pushdown Theorem). Given a functor $F: C \rightarrow D$ and a $C$-diagram $\mathscr{X}$, there is a natural homotopy equivalence

$$
\operatorname{hocolim}_{D} F_{h_{*}} \mathscr{X} \rightarrow \operatorname{hocolim}_{C} \mathscr{X} .
$$

Remark. The Homotopy Pushdown Theorem is particularly transparent if $C=P$ and $D=Q$ are poset categories. Suppose that $F: P \rightarrow Q$ is a functor (a monotone map). Then for each $q \in Q, \operatorname{Mor}_{C}(\cdot, q)=Q_{\geq q}$, the "overcategory" $q \uparrow F$ is the poset category

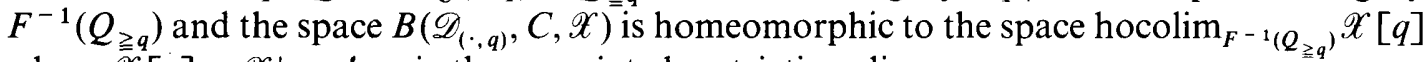
where $\mathscr{X}[q]:=\left.\mathscr{X}\right|_{F-1\left(\dot{Q}_{\geqq q}\right)}$ is the associated restriction diagram.

3.3. Homotopies arising from natural transformations. A proposition of Segal [37], Prop. 2.1 says that any natural transformation of functors $F_{0}, F_{1}: C \rightarrow D$ induces a homotopy on the level of geometric realizations. This principle has numerous consequences and applications; some of them are collected in this section.

Recall that a morphism $(F, \alpha): \mathscr{X} \rightarrow \mathscr{Y}$ of diagrams $\mathscr{X}: A \rightarrow T o p$ and $\mathscr{Y}: B \rightarrow T o p$ is a functor $F: A \rightarrow B$ and a natural transformation $\alpha$ from $\mathscr{X}$ to $\mathscr{Y} \circ F$. The result of Segal deals with topological categories and not with diagrams. However, the connection with results quoted here becomes obvious if a diagram $\mathscr{X}: A \rightarrow$ Top is viewed as a topological category with $\underset{a \in \operatorname{Obj}(A)}{\uplus} X(a)$ as the space of objects and an arrow $x \stackrel{f}{\longrightarrow} y$ for each pair of objects $x, y$ and a morphism $f \in \operatorname{Mor}_{A}(a, b)$ such that $x \in X(a), y \in X(b)$ and $X(f)(x)=y$. We are indebted to the referee for the following proposition and its corollaries. The referee also pointed out that these results can be immediately deduced from Proposition 3.1.7 in [21]; however, in the more explicit form presented here they are closer to our intended combinatorial applications.

Proposition 3.13. Suppose that $\mathscr{X}: A \rightarrow$ Top and $\mathscr{Y}: B \rightarrow$ Top are diagrams of spaces over $A$ and $B$. Assume that $(F, \alpha): \mathscr{X} \rightarrow \mathscr{Y}$ and $(G, \beta): \mathscr{Y} \rightarrow \mathscr{X}$ are morphisms of these diagrams such that either

1. there exists a natural transformation $\varepsilon: G \circ F \rightarrow$ Id such that $X(\varepsilon) \circ \beta \circ \alpha=\mathrm{id}$ or

2. a natural transformation $\bar{\varepsilon}$ : Id $\rightarrow G \circ F$ such that $\beta \circ \alpha=X(\bar{\varepsilon})$. 


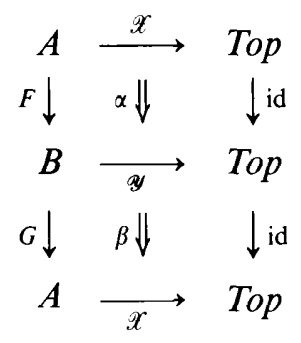

Then $(F, \alpha)$ and $(G, \beta)$ induce continuous maps

$$
f: \operatorname{hocolim}_{A} \mathscr{X} \rightarrow \operatorname{hocolim}_{B} \mathscr{Y} \text { and } g: \operatorname{hocolim}_{B} \mathscr{Y} \rightarrow \operatorname{hocolim}_{A} \mathscr{Y}
$$

such that $g$ is a left homotopy inverse to $f$,

$$
g \circ f \simeq \mathrm{Id} .
$$

Moreover, if there exists a morphism of diagrams $(H, \gamma): \mathscr{X} \rightarrow \mathscr{Y}$ and either a natural transformation $\eta: H \circ G \rightarrow$ Id such that $Y(\eta) \circ \gamma \circ \beta=$ id or a natural transformation $\bar{\eta}:$ Id $\rightarrow H \circ G$ such that $\gamma \circ \beta=Y(\bar{\eta})$, then $(F, \alpha),(G, \beta)$ and $(H, \gamma)$ induce homotopy equivalences

$$
\operatorname{hocolim}_{A} \mathscr{X} \simeq \operatorname{hocolim}_{B} \mathscr{Y} .
$$

Corollary 3.14. Let $i: B \rightarrow A$ be an inclusion of $B$ as a full subcategory of $A, F: A \rightarrow B$ a functor which maps $B$ to $B$, and $\xi: \mathrm{Id}_{A} \rightarrow i \circ F$ a natural transformation. Let $\mathscr{X}$ be an $A$-diagram, and let $\mathscr{X} \mid B$ denote the restriction of $\mathscr{X}$ to $B$. Then the canonical inclusion map induces a homotopy equivalence

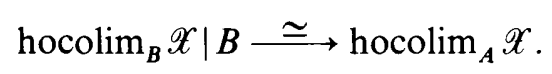

Proof. The inclusion of diagrams $\Phi: \mathscr{X} \mid B \rightarrow \mathscr{X}$ induces an inclusion map $h(\Phi): \operatorname{hocolim}_{B} \mathscr{X} \mid B \rightarrow \operatorname{hocolim}_{A} \mathscr{X}$. The natural transformation $\xi$ defines a map $\Xi: \mathscr{X} \rightarrow \mathscr{X} \mid B$ of diagrams, $a \mapsto F(a), X(a) \mapsto X \xi_{a}(a)$, which induces a map

$$
h(\Xi): \operatorname{hocolim}_{A} \mathscr{X} \rightarrow \operatorname{hocolim}_{B} \mathscr{X} \mid B .
$$

Then $h(\Phi)$ is both the right and left homotopy inverse of $h(\Xi)$. Indeed, this follows from Proposition 3.13 with $(F, \alpha)=(H, \gamma)=(F, X \xi),(G, \beta)=(i$, id $), \mathscr{Y}=\mathscr{X} \mid B=\mathscr{X} \circ i, \bar{\varepsilon}=\xi$, and $\bar{\eta}=\xi i$.

Corollary 3.15. Let $F: A \rightarrow B$ be a left adjoint to $G: B \rightarrow A$. Let $\mathscr{X}$ be an A-diagram. Then $(G, \mathrm{id})$ is a map of diagrams which induces a homotopy equivalence

$$
\operatorname{hocolim}_{B} \mathscr{X} \circ G \simeq \operatorname{hocolim}_{A} \mathscr{X} .
$$

Proof. Let $\eta:$ Id $\rightarrow G \circ F$ and $\varepsilon: F \circ G \rightarrow$ Id be the adjunction transformations. Apply Proposition 3.13 with $(F, \alpha)=(H, \gamma)=(F, \eta), Y=X \circ G,(G$, id $), \bar{\varepsilon}=\eta: \operatorname{Id} \rightarrow G \circ F$ and $\eta=\varepsilon: F \circ G \rightarrow$ Id 


\section{Diagrams over posets}

Diagrams over posets play a special role in this paper since the majority of diagrams arising in combinatorics are of this type. The situation resembles the use of diagrams of spaces in the theory of transformation groups where this technique is also of utmost importance. A topologist primarily interested in group actions may find it convenient to have the key statements formulated in the language of $G$-spaces although they are consequences of more general results. An example is the result that an equivariant map $f: X \rightarrow Y$ induces a homotopy equivalence $\hat{f}: E G \times{ }_{G} X \rightarrow E G \times{ }_{G} Y$ if $f^{H}: X^{H} \rightarrow Y^{H}$ is a homotopy equivalence for any subgroup $H \cong G[16]$.

A similar tendency exists in combinatorics. For example, the reference [34], where Propositions 4.2 and 4.3 are formulated, deals only with posets despite the fact that Quillen himself was in possession of much more general statements, his well known Theorems $A$ and B [33]. All these results are relatives of theorems formulated in Sections 3.3 and 3.2 and they have played an important role in the development of the general theory.

This is the first reason why we collect some of the corollaries of the general results in a separate section. A second reason is that there exist results which hold for posets and which do not have useful analogs for general categories.

4.1. Homotopy colimits revisited. The homotopy colimit construction is supposed to be a direct extension of the order complex construction for (locally) finite posets $P$. It is shown in Section 5.1 that the join [4], $J(\mathscr{X}):=X_{1} * \cdots * X_{n}$ of a family $\mathscr{X}=\left\{X_{i}\right\}_{1 \leqq i \leqq n}$ of spaces has a simple description as the homotopy colimit of a natural diagram of spaces. Here we note that conversely, at least in the case of posets, the homotopy colimit can be described as a subspace of a join of spaces.

Proposition 4.1. Let $\mathscr{D}: P \rightarrow$ Top be a diagram over a (locally) finite poset $P$. Let $J(\mathscr{D}):=\underset{p \in P}{*} D_{p}$ be the join of all spaces in this diagram and let

$$
\Delta(\mathscr{D}):=\left\{t_{0} x_{0}+\cdots+t_{m} x_{m} \in J(\mathscr{D}) \mid x_{i} \in D_{p_{i}}, p_{0} \leqq \cdots \leqq p_{m}, d_{p_{j+1} p_{j}}\left(x_{j+1}\right)=x_{j}\right\} .
$$

Then this space is naturally homeomorphic to the homotopy colimit of $\mathscr{D}$,

$$
\Delta(\mathscr{D}) \cong \operatorname{hocolim}_{P} \mathscr{D} .
$$

This characterization of homotopy colimits generalizes the order complex construction, since $\Delta(P) \cong \Delta\left(\mathscr{P} \mathscr{T}_{P}\right)$, where $\mathscr{P} \mathscr{T}_{P}$ is the constant diagram associated to $P$, as in Section 2 .

4.2. Comparison results for diagrams over posets. In this section we briefly review some of the most useful comparison lemmas which are meaningful only for order complexes of posets. Examples of such results are the Crosscut Theorem 4.4 and the Homotopy Complementation Formula [6], the latter one being a special case of Theorem 5.2. These results have found many interesting combinatorial applications. Also, by showing some of the most useful corollaries of general results from Section 3 we provide a link between these results and the general theory of diagrams. 
Quillen's Theorem A ([33]) and its specialization for posets the so called Fiber Theorem can be seen as a corollary and a result that motivates both the Cofinality Theorem (Proposition 3.10) and Segal's Pushdown Theorem (Proposition 3.12). The Order Homotopy Theorem is a corollary and a good illustration of results from Section 3.3.

Proposition 4.2 (Quillen Fiber Theorem: Quillen [33], [34], Walker [45]). If f: $P \rightarrow Q$ is a map of posets such that the order complex of $f^{-1}\left(Q_{\geq q}\right)$ is contractible for all $q \in Q$, then the simplicial map $\Delta f: \Delta(P) \rightarrow \Delta(Q)$ is a homotopy equivalence.

Similarly, if the order complex of $f^{-1}\left(Q_{\leqq q}\right)$ is contractible for all $q \in Q$, then $\Delta f$ is a homotopy equivalence.

Proposition 4.3 (Order Homotopy Theorem: Quillen [34], see Björner [4], (10)). If $f: P \rightarrow P$ is a decreasing poset map (that is, $f(p) \leqq p$ for all $p \in P$ ), then $P$ is homotopy equivalent to $f(P)$.

A join semilattice is a poset $P$ such that every finite subset has a unique minimal upper bound. If $P$ is of finite length, then this implies that $P$ has a unique maximal element $\hat{1}$. The crosscut complex $\Gamma(P)$ of a join semilattice is the simplicial complex of all nonempty subsets of $\min (P)$ that have an upper bound in $P \backslash \hat{1}$.

Proposition 4.4 (Crosscut Theorem: see Björner [4], (10.8)). For every join semilattice $P$ of finite length, the crosscut complex $\Gamma(P)$ is homotopy equivalent to the order complex of $P \backslash \hat{1}$.

Next we come to the combinatorial version of the Projection Lemma 3.1. The natural combinatorial setting for its application (see Sections 5.2 and 5.4) is the following: A topological space $X$ is covered by a (finite) set $\left(X_{i}\right)_{i \in I}$ of closed subspaces. The intersection poset $P$ of the covering $X=\bigcup_{i \in I} X_{i}$ is defined on the set of all spaces $\bigcap_{j \in J} X_{j}, J \subseteq I$, that occur as an intersection of spaces $\left(X_{i}\right)_{i \in I}$ with the reversed inclusion as its order relation. There is a natural diagram $\mathscr{D}: P \rightarrow$ Top associated to the covering. Namely, to each element $p \in P$ one assigns the corresponding intersection $D_{p}$ and defines the maps as the natural inclusions.

Lemma 4.5 (Projection Lemma: [3], [49]). Let $X=\bigcup_{i \in I} X_{i}$ be a covering of the space $X$ by a finite set of closed subspaces. Let $P$ be the intersection poset $P$ and let $\mathscr{D}$ be the corresponding $P$-diagram. Assume that all maps in this diagram are closed cofibrations. Then the natural map hocolim $\mathscr{D} \rightarrow \operatorname{colim} \mathscr{D} \cong X$ induces a homotopy equivalence hocolim $\mathscr{D} \cong X$.

In general a 'standard' application of the homotopy colimit tools proceeds from a diagram $\mathscr{D}$ associated to a covering to another 'more simple' diagram by suitably modifying $\mathscr{D}$ using a poset version of the Homotopy Lemma 3.7, the Cofinality Theorem 3.10 or the Upper Fiber Lemma.

Lemma 4.6 (Homotopy Lemma). Let $\mathscr{D}$ and $\mathscr{E}$ both be P-diagrams, and assume that there is a map of diagrams (id, $\alpha): \mathscr{D} \rightarrow \mathscr{E}$ such that $\alpha_{p}: D_{p} \rightarrow E_{p}$ is a homotopy equivalence for each $p \in P$, then $\alpha$ induces a homotopy equivalence $\hat{\alpha}:$ hocolim $\mathscr{D} \rightarrow$ hocolim $\mathscr{E}$. 
If one wants to change the poset underlying a diagram one needs an analog of the Cofinality Theorem 3.10 .

Lemma 4.7 (Inverse Image Lemma). Let $f: P \rightarrow Q$ be a poset morphism and let $\mathscr{E}$ be a Q-diagram. Define the P-diagram $f^{*}(\mathscr{E})$ by $\left(f^{*} E\right)_{p}:=E_{f(p)}$ and $f^{*} e_{p p^{\prime}}=e_{f(p) f\left(p^{\prime}\right)}$.

If for all $q \in Q, f^{-1}\left(Q_{\leqq q}\right)$ is contractible, then $f$ induces a homotopy equivalence

$$
\operatorname{hocolim} f^{*}(\mathscr{E}) \rightarrow \operatorname{hocolim} \mathscr{E}
$$

The following Upper Fiber Lemma is a useful result which also permits us to compare homotopy colimits of diagrams over different posets. For comparison we give two proofs of this lemma. The first and elementary proof is based on the basic principles while the second proof illustrates the use of general tools developed in earlier sections.

Lemma 4.8 (Upper Fiber Lemma). Let $\mathscr{D}$ be a P-diagram and let $\mathscr{E}$ be a $Q$-diagram. Assume $(F, \alpha): \mathscr{D} \rightarrow \mathscr{E}$ is a map of diagrams. If $(F, \alpha)$ induces a homotopy equivalence of the restrictions

$$
\hat{\alpha}:\left.\operatorname{họcolim} \mathscr{D}\right|_{p \in P: F(p) \geqq q} \simeq \text { hocolim }\left.\mathscr{E}\right|_{Q_{\geqq q}} \text { for all } q \in Q,
$$

then $(F, \alpha)$ induces a homotopy equivalence $\hat{\alpha}:$ hocolim $\mathscr{D} \rightarrow$ hocolim $\mathscr{E}$.

Proof. ( $1^{\text {st }}$ proof) We proceed by induction on the cardinality $|Q|$ of $Q$. Let $q \in Q$ be a minimal element of $Q$. Then $Q=Q_{1} \cup Q_{2}$ for $Q_{1}=Q_{\geq q}$ and $Q_{2}=Q \backslash\{q\}$. The poset $Q_{3}:=Q_{1} \cap Q_{2}$ equals $Q_{>q}$. We set $P_{i}:=f^{-1}\left(Q_{i}\right)$ for $i=1,2,3$, which implies $P_{1} \cap P_{2}=P_{3}$ and $P_{1} \cup P_{2}=P$.

If $q$ is the unique minimal element of $Q$ (i.e., $Q_{1}=Q$ ), then the assertion follows trivially from the assumptions. Otherwise $\alpha$ induces a homotopy equivalence $\hat{\alpha}:\left.\operatorname{holim} \mathscr{D}\right|_{P_{i}} \rightarrow$ hocolim $\left.\mathscr{E}\right|_{Q_{i}}$ for $i=1,2,3$, by induction. Since

and

$$
\text { hocolim }\left.\mathscr{D}\right|_{P_{1}} \cap \text { hocolim }\left.\mathscr{D}\right|_{P_{2}}=\text { hocolim }\left.\mathscr{D}\right|_{P_{3}}
$$

$$
\left.\operatorname{hocolim} \mathscr{D}\right|_{Q_{1}} \cap \text { hocolim }\left.\mathscr{D}\right|_{Q_{2}}=\left.\operatorname{hocolim} \mathscr{D}\right|_{Q_{3}} \text {, }
$$

it follows from the Gluing Lemma 2.4 that $\alpha$ induces a homotopy equivalence between

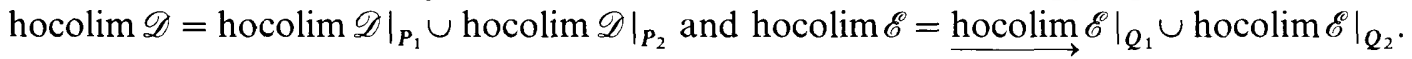

( $2^{\text {nd }}$ proof) By the Homotopy Pushdown Theorem, hocolim $\mathscr{D}_{P} \simeq \operatorname{hocolim}_{Q} F_{h_{*}}(\mathscr{D})$. By the remark after the Proposition 3.12, $F_{h_{*}}(\mathscr{D}): Q \rightarrow T o p$ is the diagram defined by $F_{h_{*}}(\mathscr{D})(q):=\left.\operatorname{hocolim}_{F^{-1}\left(Q_{\underline{z}}\right)} \mathscr{D}\right|_{F^{-1}}\left(Q_{\geq q}\right)$. Let $\mathscr{E}^{\prime}: Q \rightarrow T o p$ be the diagram defined by $\mathscr{E}^{\prime}(q):=\left.\operatorname{hocolim}_{Q_{\geq q}} \mathscr{E}\right|_{Q_{\geq q}}$. Then the natural map $\mathscr{E}^{\prime}(q) \rightarrow \mathscr{E}(q)$ is a homotopy equivalence by Corollary 3.14. Finally, since by assumption the natural map $F_{h_{*}}(\mathscr{D})(q) \rightarrow \mathscr{E}^{\prime}(q)$ is a homotopy equivalence, the proposition follows from the Homotopy Lemma, Proposition 3.7 .

If one is lucky - and surprisingly often one is indeed lucky - one is able to transform a diagram $\mathscr{D}$ into a diagram that allows an application of the Wedge Lemma 3.5 whose poset version we state next. 
Lemma 4.9 (Wedge Lemma). Let $P$ be a poset with maximal element $\hat{1}$. Let $\mathscr{D}$ be a $P$-diagram so that there exist points $c_{p^{\prime}} \in D_{p^{\prime}}$ for all $p^{\prime}<\hat{1}$ such that $d_{p p^{\prime}}$ is the constant map $d_{p p^{\prime}}: x \mapsto c_{p^{\prime}}$, for $p>p^{\prime}$. Then

$$
\operatorname{hocolim} \mathscr{D} \simeq \bigvee_{p \in P}\left(\Delta\left(P_{<p}\right) * D_{p}\right)
$$

where the wedge is formed by identifying $c_{p} \in \Delta\left(P_{<p}\right) * D_{p}$ with $p \in \Delta\left(P_{<\hat{1}}\right) * D_{\hat{1}}$, for $p<\hat{1}$.

We close this toolkit of lemmas by a result that is a simple consequence of the definition of a homotopy colimit.

Lemma 4.10 (Embedding Lemma). Let (id, $\alpha): \mathscr{D} \rightarrow \mathscr{E}$ be a map of P-diagrams. If $\alpha: D_{p} \hookrightarrow E_{p}$ is a closed embedding for every $p \in P$, then $\alpha$ induces a closed embedding

$$
\hat{\alpha}: \text { hocolim } \mathscr{D} \hookrightarrow \text { hocolim } \mathscr{E} .
$$

In particular, if $\mathscr{D}$ is a $P$-diagram and $Q \subseteq P$ is a subposet, then hocolim $\mathscr{D} \mid Q \hookrightarrow$ hocolim $\mathscr{D}$ is a closed embedding.

\section{Applications}

5.1. Combinatorial objects as homotopy colimits. In this section we show how some well known combinatorial constructions can be usefully related to diagrams and their homotopy colimits.

Proposition 5.1. Let $\mathscr{X}=\left\{X_{i}\right\}_{i=0}^{n}$ be a collection of spaces. Denote by $\mathscr{B}_{n}$ the poset of all nonempty faces of an n-simplex, i.e., the poset $2^{\{1, \ldots, n+1\}} \backslash\{\emptyset\}$ ordered by inclusion. Let $\mathscr{D}_{\mathscr{X}}: \mathscr{B}_{n} \rightarrow$ Top be the following diagram associated to the collection $\mathscr{X}$. For $A \in \mathscr{B}_{n}$ let $\mathscr{D}_{\mathscr{X}}(A):=\prod_{i \in A} X_{i}$ and for $A \supseteq B$ let $d_{A B}: \mathscr{D}_{\mathscr{X}}(A) \rightarrow \mathscr{D}_{\mathscr{X}}(B)$ be the canonical projection. Then the join $J(\mathscr{X})$ of all spaces in the family $\mathscr{X}$ is naturally homeomorphic to the homotopy colimit of this diagram

$$
J(\mathscr{X}):=X_{0} * X_{1} * \cdots * X_{n} \cong \operatorname{hocolim}_{\mathscr{B}_{n}} \mathscr{D}_{\mathscr{X}} .
$$

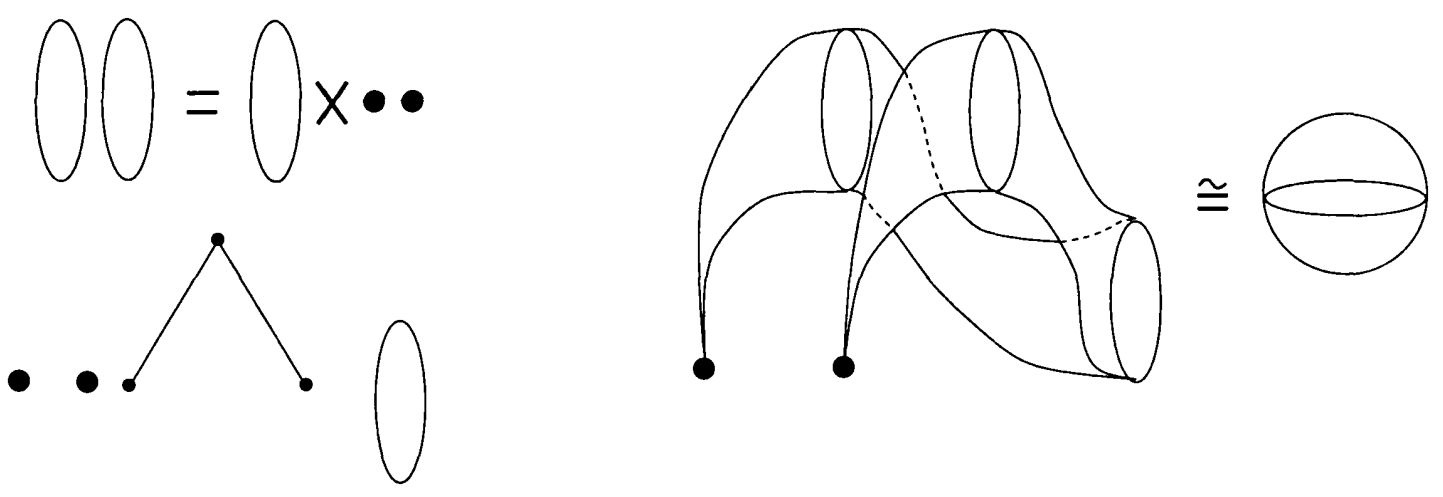

Figure 1. The join of $S^{0}$ and $S^{1}$ as a homotopy colimit 
The formula given in Proposition 5.1 is a very simple and useful representation of the join of spaces exposing much of the underlying combinatorial structure.

An important variation of the diagram above arises if $X_{i}=S^{1}$ for all $i$. This is a toric diagram in the sense of Section 5.3. There is a natural diagonal action of the circle group $U(1)$ on all tori $\mathscr{D}(A)=\left(S^{1}\right)^{|A|}$. Dividing by this action leads to a new diagram $\mathscr{E}:=\mathscr{D} / U(1)$ and a well known fact is that this leads to a combinatorial description of the complex projective space $\mathbb{C} P^{n}$ :

$$
\operatorname{hocolim}_{\mathscr{B}_{n}} \mathscr{E} \cong \mathbb{C} P^{n}
$$

A very useful concept, which has found numerous applications in combinatorics, is the deleted join operation [4], [35], [50]. Given a simplicial complex $K$, the $p$ th deleted join $K_{\delta}^{*(p)}$ is the subcomplex of the join $K^{*(p)}:=K * \cdots * K$ of $p$ copies of $K$ which consists of all simplices $\theta \in K^{*(p)}$ which have the form $\theta=\theta_{1} * \cdots * \theta_{p}$ where $\theta_{i}, i=1, \ldots, p$, are pairwise vertex disjoint simplices of $K$. Some very important complexes can be constructed by iterating the join and the deleted join operation, for example the well known chessboard complexes $\Delta_{p, q}[4]$, [50], arise this way. The simplest complex of this form is $J_{N, q}:=\left(\Delta^{N}\right)_{\delta}^{*(q)}$, where $\Delta^{N}$ is the standard $N$-dimensional simplex. This space, viewed as a space with the obvious cyclic $\mathbb{Z}_{q}$-action, has been applied in combinatorics to problems of Tverberg type, to the 'necklace' partition problem, etc., see [4]. Motivated by these and other similar examples, it is natural to look for homotopy colimit representations for deleted joins as well.

There are two obvious ways to associate a diagram to the space of the form $K_{\delta}^{*(p)}$. One can view $K_{\delta}^{*(p)}$ as a subspace of $K^{*(p)}$ which has the homotopy colimit representation described in Proposition 5.1. The subdiagram which arises this way is similar except that the products $K^{j}$ are replaced by deleted products $K_{\delta}^{j}, j=1, \ldots, p$. Recall that the deleted product $K_{\delta}^{j}$ is a cell subcomplex of the cell complex $K^{j}$ consisting of all cells of the form $\theta=\theta_{1} \times \cdots \times \theta_{j}$, where $\theta_{k}, k=1, \ldots, j$, are pairwise vertex disjoint simplices in $K$. Another possibility is to link $K_{\delta}^{*(p)}$ with a diagram over the face poset of $K$ itself via the 'collapsing' map $K_{\delta}^{*(p)} \rightarrow K$. The diagram arising this way is a finite space diagram. For example if $K=\Delta^{N}$, the space $K_{\delta}^{*(q)}=J_{N, q}$ is the homotopy colimit of a diagram of the type described in Proposition 5.1 where $X_{i}:=[q] \cong \mathbb{Z}_{q}$ for all $i=0,1, \ldots, N$. These and other examples indicate that it may be useful to study discrete analogs of toric diagrams with the discrete 'tori' $\mathbb{Z}_{q}^{j}=\mathbb{Z}_{q} \times \cdots \times \mathbb{Z}_{q}$ in place of $T^{j}=S^{1} \times \cdots \times S^{1}$. Specially it is an interesting question which convex polytopes admit natural discrete toric diagrams.

5.2. Björner's generalized homotopy complementation formula. Björner's generalized homotopy complementation formula [5] is an effective tool to compute the homotopy type of a simplicial complex $\Delta$ in the case when a large, contractible induced subcomplex $\Delta_{\bar{A}}$ is known, whose connections to the rest of the complex are not too complicated.

In the following, we provide a "diagrammatic" proof of Björner's result, thereby demonstrating the applicability of some of our lemmas.

Let $\Delta \cong 2^{S}$ be a finite (abstract) simplicial complex with vertex set $S$, let $A \cong S$ be a subset of its vertex set, and denote by $\bar{A}$ the complement $S \backslash A$ of $A$. 
Let $\Delta_{A}:=\{\sigma \in \Delta: \sigma \subseteq A\}=\Delta \cap 2^{A}$ be the induced subcomplex on $A$, and similarly $\Delta_{\bar{A}}$ the induced complex on $\bar{A}$. In the following, the key assumption we will make is that $\Delta_{\bar{A}}$ is contractible.

Theorem 5.2 (Generalized homotopy complementation formula: Björner [5]). For any simplicial complex $\Delta \subseteq 2^{S}$ and $A \subseteq S$, define a new simplicial complex $T_{A}$ by taking the union of all the simplicial complexes

$$
(p \uplus \sigma) *\left(\operatorname{star}_{\Delta}(\sigma) \cap \Delta_{\bar{A}}\right) \quad \text { for } \sigma \in \Delta,
$$

where $p$ is an additional point $p \notin S$, and $*$ denotes the join of two complexes.

If $\Delta_{\bar{A}}$ is contractible, then $\Delta$ and $T_{A}$ are homotopy equivalent.

Proof. Let $P$ be the poset of all nonempty faces of $\Delta$, ordered by inclusion, and let

$$
Q:=\{(\sigma, \tau) \in P \times P: \sigma \leqq \tau\}
$$

partially ordered by the condition

$$
\left(\sigma^{\prime}, \tau^{\prime}\right) \leqq(\sigma, \tau): \Leftrightarrow \sigma \leqq \sigma^{\prime} \leqq \tau^{\prime} \leqq \tau
$$

(Thus $Q$ is isomorphic to the poset of all intervals in $P$, ordered by inclusion: $Q \cong \operatorname{Int}(P)$.)

For $\sigma \in P$ we define

$$
D_{\sigma}:=\operatorname{star}_{4}(\sigma) \cup \Delta_{\bar{A}}
$$

Then we clearly have inclusion maps $d_{\tau \sigma}: D_{\tau} \hookrightarrow D_{\sigma}$ whenever $\tau \geqq \sigma$. This defines a $P$ diagram $\mathscr{D}$, with

$$
\Delta=\operatorname{colim} \mathscr{D} \simeq \text { hocolim } \mathscr{D},
$$

where the equality holds by definition of the colimit (this is a subspace diagram!), and the homotopy equivalence is an application of the Projection Lemma 4.5.

Similarly, for $(\sigma, \tau) \in Q$ we define

$$
E_{(\sigma, \tau)}:=(p \uplus \sigma) *\left(\operatorname{star}_{\Delta}(\tau) \cap \Delta_{\bar{A}}\right) .
$$

Then we get inclusion maps $e_{(\sigma, \tau)\left(\sigma^{\prime}, \tau^{\prime}\right)}: E_{(\sigma, \tau)} \hookrightarrow E_{\left(\sigma^{\prime}, \tau^{\prime}\right)}$ whenever $(\sigma, \tau) \geqq\left(\sigma^{\prime}, \tau^{\prime}\right)$. This defines a $Q$-diagram $\mathscr{E}$, with

$$
T_{A}=\operatorname{colim} \mathscr{E} \simeq \text { hocolim } \mathscr{E},
$$

by definition of the colimit and the Projection Lemma.

Thus, the claim of the theorem is reduced to proving that the homotopy colimits of the $P$-diagram $\mathscr{D}$ and the $Q$-diagram $\mathscr{E}$ are homotopy equivalent. This demands use of our new homotopy lemmas, since the posets $P$ and $Q$ are quite different. 
The canonical poset map to use is

$$
f: Q \rightarrow P, \quad(\sigma, \tau) \rightarrow \tau
$$

This $f$ induces a homotopy equivalence between the posets $P$ and $Q$ (Walker [46]). To see this, we observe that the lower fibers $f^{-1}\left(P_{\leqq \tau}\right)$ are canonically isomorphic to $\operatorname{Int}\left(P_{\leqq \tau}\right)$. On the poset $\operatorname{Int}\left(P_{\leqq \tau}\right)$ we have an increasing map $g: \operatorname{Int}\left(P_{\leqq \tau}\right) \rightarrow \operatorname{Int}\left(P_{\leq_{\tau}}\right)$ given by $\left(\sigma^{\prime}, \tau^{\prime}\right) \mapsto\left(\sigma^{\prime}, \tau\right)$. Thus, by the Order Homotopy Theorem 4.3 the fiber $\operatorname{Int}\left(P_{\leqq \tau}\right)$ is homotopy equivalent to the image $g\left(\operatorname{Int}\left(P_{\leqq \tau}\right)\right) \cong P_{\leqq \tau}$, which is a cone.

Now we modify $\mathscr{E}$ and $\mathscr{D}$ a little. We define a new $Q$-diagram $\mathscr{E}^{\prime}$, whose spaces are

$$
E_{(\sigma, \tau)}^{\prime}:=\operatorname{star}_{\Delta}(\tau) \cup\left(p * \Delta_{\bar{A}}\right),
$$

and whose maps are the obvious inclusions. Furthermore, there is a map $\psi_{1}: \mathscr{E} \rightarrow \mathscr{E}$, which is the identity map on $Q$, and between the spaces uses the inclusion maps

$$
(p \uplus \sigma) *\left(\operatorname{star}_{\Delta}(\tau) \cap \Delta_{\bar{A}}\right) \hookrightarrow(p \uplus \tau) *\left(\operatorname{star}_{\Delta}(\tau) \cap \Delta_{\bar{A}}\right) \hookrightarrow \operatorname{star}_{\Delta}(\tau) \cup\left(p * \Delta_{\bar{A}}\right)
$$

which are clearly homotopy equivalences, since $\Delta_{\bar{A}}$ is contractible. Thus, by the Homotopy Lemma $4.6, \psi_{1}$ induces a homotopy equivalence

$$
\hat{\psi}_{1}: \operatorname{hocolim} \mathscr{E} \simeq \text { hocolim } \mathscr{E}^{\prime} .
$$

Similarly, we define a new $P$-diagram $\mathscr{D}^{\prime}$, whose spaces are

$$
D_{\tau}^{\prime}:=\operatorname{star}_{\Delta}(\tau) \cup\left(p * \Delta_{\bar{A}}\right)
$$

and whose maps are the obvious inclusions. Furthermore, there is a map $\psi_{2}: \mathscr{D} \rightarrow \mathscr{D}^{\prime}$, which is the identity map on $P$, and between the spaces uses the inclusion maps

$$
\operatorname{star}_{\Delta}(\tau) \cup \Delta_{\bar{A}} \hookrightarrow \operatorname{star}_{\Delta}(\tau) \cup\left(p * \Delta_{\bar{A}}\right)
$$

which are clearly homotopy equivalences. Thus, by the Homotopy Lemma, $\psi_{2}$ induces a homotopy equivalence

$$
\hat{\psi}_{2}: \operatorname{hocolim} \mathscr{D} \simeq \operatorname{hocolim} \mathscr{D}^{\prime}
$$

Finally, at this stage we see that $\mathscr{E}^{\prime}$ is an inverse image diagram, $\mathscr{E}^{\prime}=f^{*}\left(\mathscr{D}^{\prime}\right)$, where $f$ is a poset map whose lower fibers we have already checked to be contractible. Hence the Inverse Image Lemma 4.7 implies a homotopy equivalence

$$
\text { hocolim } \mathscr{E}^{\prime} \simeq \operatorname{hocolim} \mathscr{D}^{\prime}
$$

which completes the proof.

Alternatively, one could derive hocolim $\mathscr{E}^{\prime} \simeq$ hocolim $\mathscr{D}^{\prime}$ also from the Upper Fiber Lemma 4.8, together with the Projection Lemma 4.5, since $\mathscr{E}^{\prime}$ and $\mathscr{D}^{\prime}$ are subspace diagrams as well. 
We note that there are alternative ways to describe the construction of $T_{A}$. For example, one can (as Björner does in [5]) start from a wedge (or a disjoint union) of the spaces $E_{(\sigma, \sigma)}=(p \uplus \sigma) *\left(\operatorname{star}_{\Delta}(\sigma) \cap \Delta_{\bar{A}}\right)$, and then check that all the identifications of the colimit colim $\mathscr{E}$ are generated by identifying, for $\sigma \subset \tau$, the identical subcomplexes $(p \uplus \sigma) *\left(\operatorname{star}_{\Delta}(\tau) \cap \Delta_{\bar{A}}\right)$ in $E_{(\sigma, \sigma)}$ and in $E_{(\tau, \tau)}$.

Also, there are countless variations possible, corresponding to different coverings of the complex $\Delta$. The beauty of Björner's set-up is that his transformations of $\Delta$ end up with a subspace diagram, and thus with a colimit instead of a homotopy colimit, which leads to an effective model for $\Delta / \Delta_{\bar{A}}$. It seems to us that the diagram techniques yield an extremely natural and convenient setting for the proof of the generalized homotopy complementation formula and similar results.

5.3. Toric varieties. In this section we give a representation of the topological space underlying a toric variety (see Danilov [9], Fulton [18], and Ewald [13] for general background on toric varieties) as the homotopy colimit of a diagram. For this we recall a description, due to MacPherson (see Yavin and Fischli [48], [17]), of a toric variety. A decomposition of $\mathbb{R}^{n}$ into a complex $\Sigma$ of closed, convex, polyhedral cones with apex $\mathbf{0}$ is .called a complete fan. If all cones in $\Sigma$ are generated by lattice points in $\mathbb{Z}^{n}$, then $\Sigma$ is called rational. Assume that $\Sigma$ is a complete and rational fan in $\mathbb{R}^{n}$. Then let $\mathscr{P}$ be the cell decomposition of the unit ball in $\mathbb{R}^{n}$ that is dual to the one induced by $\Sigma$. For $\sigma \in \Sigma$ we denote by $\check{\sigma}$ the cell in $\mathscr{P}$ that corresponds to $\sigma$. Thus $\check{\sigma}$ is a cell of dimension $n-\operatorname{dim}(\sigma)$.

We identify the $n$-torus $\mathscr{T}^{n}$ with the image of the projection map $\pi: \mathbb{R}^{n} \rightarrow \mathbb{R}^{n} / \mathbb{Z}^{n}$. For all cones $\sigma \in \Sigma$ the image of $\sigma$ under this projection is a subtorus $\pi(\sigma)=\pi\left(\operatorname{span}_{\mathbb{R}}(\sigma)\right)=\mathscr{T}$ of $\mathscr{T}$. Since $\sigma$ is rational, this is a closed subtorus of dimension $\operatorname{dim}(\sigma)$. Thus the quotient $\mathscr{T}^{n} / \mathscr{T}_{\sigma}$ is a real torus of dimension $n-\operatorname{dim}(\sigma)$.

The toric variety $X_{\Sigma}$ is obtained from $\mathscr{P} \times \mathscr{T}^{n}$ by taking the quotient of $(\check{\sigma})^{\circ} \times \mathscr{T}^{n}$ by the action of $\mathscr{T}$ on $\mathscr{T}$ for each $\sigma \in \Sigma$. This leads to a nice (compact, Hausdorff) quotient space since we take quotients by larger tori on $\partial \sigma \times \mathscr{T}^{n}$. In particular, we see that the toric variety $X_{\Sigma}$ has a well-defined map $\Pi: X_{\Sigma} \rightarrow \mathscr{P}$, for which the fiber over any interior point of $\check{\sigma}$ is isomorphic to $\mathscr{T} / \mathscr{T}_{\sigma}$.

Let $P_{\Sigma}$ be the poset whose elements are in bijection with the cones in $\Sigma$ and whose order relation is defined by reversed inclusion of the cones in $\Sigma$. Thus $P_{\Sigma}$ is the poset of non-empty faces of $\mathscr{P}$, ordered by inclusion. In particular, $P_{\Sigma}$ has a largest element $\hat{1}$ corresponding to the 0 -dimensional cone $\{\boldsymbol{0}\}$. We construct a diagram $D_{\Sigma}$ over the poset $P_{\Sigma}$ as follows. For $\sigma \in \Sigma$, set $D_{\sigma}=\mathscr{T} / \mathscr{T}_{\sigma}$. Topologically, $D_{\sigma}$ is an $(n-\operatorname{dim}(\sigma))$-torus. The map $d_{\tau, \sigma}$ for $\tau \leqq \sigma$ is the map induced by the projection $\mathscr{T} / \mathscr{T}_{r} \rightarrow \mathscr{T} / \mathscr{T}_{\sigma}$.

Proposition 5.3. Let $\Sigma$ be a complete and rational fan in $\mathbb{R}^{n}$. Then the toric variety $X_{\Sigma}$ is homeomorphic to the homotopy colimit of the diagram $D_{\Sigma}$ associated with $\Sigma$ :

$$
\operatorname{hocolim} D_{\Sigma} \cong X_{\Sigma} .
$$

Proof. Let $\phi$ be the map that sends $\mathscr{T} / \mathscr{T}_{\sigma} \times P_{\leq \sigma}$ to its image in $X_{\Sigma}$. By construction of $D_{\Sigma}$ the map $\phi$ is compatible with the equivalence relation $\equiv$ on $\underset{\sigma \in P}{\uplus} \mathscr{T} / \mathscr{T}_{\sigma} \times P_{\leqq \sigma}$. Hence 
$\phi$ induces a map $\hat{\phi}:$ hocolim $D_{\Sigma} \rightarrow X_{\Sigma}$. It is routine to check that $\hat{\phi}$ is indeed a homeomorphism.

The resolution of singularities of a toric variety also fits our homotopy colimit framework. Namely, let $\Sigma^{\prime}, \Sigma$ be two complete, rational fans in $\mathbb{R}^{n}$ such that $\Sigma^{\prime}$ is a refinement of $\Sigma$ (i.e., for every open cone $\tau^{\prime \circ}$ in $\Sigma^{\prime}$ there is an open cone $\tau^{\circ}$ in $\Sigma$ such that $\tau^{\prime \circ} \cong \tau^{\circ}$ ). Thus there is an induced map $f: P_{\Sigma^{\prime}} \rightarrow P_{\Sigma}$. Also assume that $\tau^{\prime}$ is a cone in $\Sigma^{\prime}$ whose interior is contained in the interior of the cone $\tau$ of $\Sigma$. Then the inclusion $\tau^{\prime} \hookrightarrow \tau$ induces a surjective map $\phi_{\tau^{\prime} \tau}: \mathscr{T} / \mathscr{T}_{\tau^{\prime}} \rightarrow \mathscr{T} / \mathscr{T}_{\tau}$. It is easily seen that $\phi$ induces a map of diagrams. Hence there is an induced map $\hat{\phi}$ : hocolim $D_{\Sigma^{\prime}} \cong X_{\Sigma^{\prime}} \rightarrow$ hocolim $D_{\Sigma} \cong X_{\Sigma}$. The map $\hat{\phi}$ is surjective since $f$ and all $\phi_{\tau^{\prime} \tau}$ are surjective.

Proposition 5.4. Let $\Sigma^{\prime}$ be a complete rational fan which is the refinement of the complete rational fan $\Sigma$. Then there is a surjective map $\hat{\phi}: X_{\Sigma^{\prime}} \rightarrow X_{\Sigma}$.

It is well known that $X_{\Sigma}$ is non-singular if and only if $\Sigma$ is simplicial (i.e., all cones are simplicial) and unimodular (i.e., all full-dimensional cones are equivalent to $\left\{x \in \mathbb{R}^{n}: x \geqq \mathbf{0}\right\}$ under unimodular transformations from $\left.\mathrm{GL}\left(\mathbb{R}^{n}, \mathbb{Z}\right)\right)$. For an example that shows that $H^{*}\left(X_{\Sigma} ; \mathbb{Q}\right)$ is not a combinatorial invariant of $\Sigma$ in general see [28]. It is also well known that for any complete rational fan $\Sigma$ there is a simplicial and unimodular complete rational fan $\Sigma^{\prime}$ which refines $\Sigma$. In this case $\hat{\phi}$ is a resolution of singularities.

One can also use our results to investigate the (co)homology of a toric variety. For this we set up a spectral sequence introduced by Segal [37], which uses the filtration of hocolim $D$ by the $s$-skeleta of the order complexes. For a simplicial complex $\Delta$ we denote by $\Delta^{s}$ its $s$-skeleton.

Assume $D$ is a $P$-diagram for a poset $P$. Then we denote by hocolim $D^{s}$ the image of $\uplus_{p \in P} D_{p} \times \Delta\left(P_{\leqq p}\right)^{s}$ in hocolim $D$. The filtration hocolim $D^{0} \subseteq$ hocolim $D^{1} \subseteq \cdots \subseteq$ hocolim $D$ defines a spectral sequence with termination at $\tilde{H}_{*}($ hocolim $D)$ in the $E^{2}$-term and $E_{s t}^{1}=\tilde{H}_{s+t}\left(\right.$ hocolim $D^{s}$, hocolim $\left.D^{s-1}\right)$. Following Segal's arguments one finds that $E_{s t}^{1}$ is given by $\bigoplus_{\sigma_{0}<\cdots<\sigma_{s} \in \Delta(P)} \tilde{H}_{t}\left(\mathscr{T} / \mathscr{T}_{\sigma_{s}}\right)$. Now assume $\left(\sigma_{0}<\cdots<\sigma_{s}\right) \times c$ is a $(s+t)$-cell in $\operatorname{hocolim} D^{s}$. Then the differential of the cell complex hocolim $D$ is given by

$$
\begin{gathered}
\partial\left(\sigma_{0}<\cdots<\sigma_{s}\right) \times c=\sum_{i=0}^{s-1}(-1)^{i}\left(\sigma_{0}<\cdots<\hat{\sigma}_{i}<\cdots<\sigma_{s}\right) \times c \\
+(-1)^{s-1}\left(\sigma_{0}<\cdots<\sigma_{s-1}\right) \times d_{\sigma_{s} \sigma_{s-1}}(c)+(-1)^{s}\left(\sigma_{0}<\cdots<\sigma_{s}\right) \times \partial c .
\end{gathered}
$$

Thus the differential $d_{s t}^{1}: E_{s t}^{1} \rightarrow E_{s-1, t}^{1}$ applied to the cell $\left(\sigma_{0}<\cdots<\sigma_{s}\right) \times c$ equals $\sum_{i=0}^{s-1}(-1)^{i}\left(\sigma_{0}<\cdots<\hat{\sigma}_{i}<\cdots<\sigma_{s}\right) \times c+(-1)^{s-1}\left(\sigma_{0}<\cdots<\sigma_{s-1}\right) \times d_{\sigma_{s} \sigma_{s-1}}(c)$, where $c$ is a cycle in $H_{t}\left(\mathscr{T} / \mathscr{T}_{\sigma_{s}}\right)$.

From this it is easily seen that our spectral sequence is isomorphic to the deRahmHodge spectral sequence applied by Danilov [9], Chap. 3, §12, to compute the cohomology of a toric variety. 
5.4. Subspace arrangements. Arrangements of affine subspaces in $\mathbb{R}^{n}$ also allow an application of the homotopy colimit method. Let $A$ be a finite set of affine subspaces in $\mathbb{R}^{n}$. Let us denote by $\hat{A}$ the corresponding arrangement of spheres in the one-point compactification $S^{n}$ of $\mathbb{R}^{n}$. Under our assumptions intersections of spheres in $\hat{A}$ are again spheres (or the compactification point). The following result can be deduced from the Projection Lemma 4.5, the Homotopy Lemma 4.6, and the Wedge Lemma 4.9.

Theorem 5.5 (Ziegler and Živaljević [49]). Let A be a finite set of affine subspaces in $\mathbb{R}^{n}$. Let $\widehat{U}_{A}$ be the one-point compactification of the set-theoretic union of the subspaces in $A$ and let $P$ be the intersection poset of $A$. Then

$$
\widehat{U_{A}} \simeq \bigvee_{p \in P} S^{\operatorname{dim}(p)} * \Delta\left(P_{<p}\right)
$$

An equivalent result can be found in Vassiliev [43], III. $\S 6$, Thm.1. In Vassiliev's formulation the spaces $\Delta\left(P_{<p}\right)$ are replaced by quotients of simplices by crosscut complexes, the spaces $K(p)$ in his notation. More precisely, for an arbitrary subspace $V$ corresponding to some point $p=p_{V}$ in the intersection lattice $P$ of $A$, let $V_{1}, \ldots, V_{t}$ be the subspaces in $A$ such that $V_{i}$ contains $V$ as a subspace. Let $\Sigma(p)$ be the simplex which is spanned, in the abstract sense, by the vertices $V_{1}, \ldots, V_{t}$. Vassiliev calls a face $\tau$ of $\Sigma(p)$ marginal if $V$ is $n o t$ the intersection of the subspaces corresponding to the vertices of $\tau$. Thus the marginal faces are the simplices in the crosscut complex $\Gamma\left(P_{\leqq p}\right)$ of $P_{\leqq p}$. By the Crosscut Theorem 4.4 the complex of marginal faces is homotopy equivalent to $\Delta\left(P_{<p}\right)$. In Vassiliev's formula the spaces $S^{\operatorname{dim}(p)} * \Delta\left(P_{<p}\right)$ are replaced by $S^{\operatorname{dim}(p)-1} * \Sigma(p) / \Gamma\left(P_{\leqq p}\right)$. Let us analyze $\Sigma(p) / \Gamma\left(P_{\leqq p}\right)$. If $\Gamma\left(P_{\leqq p}\right)$ is the full simplex $\Sigma(p)$, then $\Sigma(p) / \Gamma\left(P_{\leqq p}\right)$ and by the Crosscut Theorem also $\Delta\left(P_{<p}\right)$ are contractible. In particular,

$$
S^{\operatorname{dim}(p)} * \Delta\left(P_{<p}\right) \quad \text { and } \quad S^{\operatorname{dim}(p)-1} * \Sigma(p) / \Gamma\left(P_{\leqq p}\right)
$$

are contractible. If $\Gamma\left(P_{\leqq p}\right)$ is some non-empty part of the boundary of $\Sigma(p)$ then $\Sigma(p) / \Gamma\left(P_{\leqq p}\right)$ is the suspension of $\Gamma\left(P_{\leqq p}\right)$. Thus again the Crosscut Theorem shows that

$$
S^{\operatorname{dim}(p)} * \Delta\left(P_{<p}\right) \quad \text { and } \quad S^{\operatorname{dim}(p)-1} * \Sigma(p) / \Gamma\left(P_{\leqq p}\right)
$$

are homotopic. If $\Gamma\left(P_{\leqq p}\right)$ is empty, then we have to "interpret" $\Sigma(p) / \Gamma\left(P_{\leqq p}\right)$ as the suspension of the empty space, which is in our definition the join with a two point space. Then the homotopy equivalence also follows in this case.

By Alexander duality on $S^{n}$ we infer from Theorem 5.5 the following formula of Goresky and MacPherson [20].

Corollary 5.6 (Goresky and MacPherson [20]). Let $A$ be a finite set of affine subspaces in $\mathbb{R}^{n}$. Let $M_{A}$ be the complement $S^{n}-\widehat{U_{A}}$ and let $P$ be the intersection poset of $A$. Then

$$
\tilde{H}^{i}\left(M_{A} ; \mathbb{Z}\right) \cong \bigoplus_{p \in P} \tilde{H}_{\operatorname{codim}(p)-i-2}\left(\Delta\left(P_{<p}\right) ; \mathbb{Z}\right),
$$

where $\operatorname{codim}(p)$ denotes the real codimension of the subspace corresponding to $p$. 
Analogous results for arrangements of spheres and projective spaces can be found in Goresky and MacPherson [20] and in [49]. In the following, we describe a simpler, more general, and more powerful approach that provides combinatorial formulas for quite general "Grassmannian arrangements". Let $A$ be a central arrangement in $\mathbb{R}^{n}$ (or $\mathbb{C}^{n}, \mathbb{H}^{n}$ ) with intersection poset $P$ and a dimension function $d: P \rightarrow \mathbb{N}_{0}$. Let $\mathscr{D}=\mathscr{D}(A)=\left\{A_{p}\right\}_{p \in P}$ be the corresponding $P$-diagram of linear spaces. In case each of the linear subspaces $A_{p}$, $p \in P$, is invariant under the action of a finite (or just closed) subgroup $G \subset \mathrm{O}(n, \mathbb{R})$ (resp. $\mathrm{U}(n), \mathrm{Sp}(n))$ of the orthogonal (unitary, symplectic) group, a natural step to make is to define the associated orbit diagram. More generally, if $T$ is an operation (a functor) associating a space $T(V)$ to a linear subspace $V \subset \mathbb{K}^{n}$, where $\mathbb{K}=\mathbb{R}, \mathbb{C}$ or $\mathbb{H}$, then $T(A)$ denotes the diagram $T(A)=\left\{T\left(A_{p}\right)\right\}_{p \in P}$ associated to the corresponding arrangement of subspaces in $T\left(\mathbb{K}^{n}\right)$. There are several examples that come up very naturally in the mathematical practice. For example, if $V \mapsto S(V)$ is the operation of associating the unit sphere to the linear subspace $V \subset \mathbb{R}^{n}$, then $S(A)=\left\{S\left(A_{p}\right)\right\}_{p \in P}$ is the associated spherical diagram. Similarly, functors $V \mapsto \mathbb{R} P(V), V \mapsto \mathbb{C} P(V)$ or simply $P(V)$ in both cases (and in the case of quaternionic spaces) lead to the corresponding projective arrangements $\mathbb{R} P(A), \mathbb{C} P(A)$ or $\mathbb{H} P(A)$, denoted simply by $P(A)$. Projective diagrams are special cases of the associated Grassmann diagrams obtained with the aid of the functor

$$
V \mapsto G_{k}(V):=\{L \subset V: \operatorname{dim}(V)=k\} .
$$

Lens space arrangements $L_{m}(A)$ are defined similarly, where $L_{m}(V)=L_{m}(S(V)):=S(V) / \mathbb{Z}_{m}$ is the lens space associated to the unit sphere in a complex linear space $V$.

We illustrate how the Homotopy Lemma 4.6 can be applied to produce a combinatorial description of the link of the related arrangement in all the special cases above. We obtain in particular simpler, stronger, and more natural proofs of some results from [49], Thms. 2.11 and 2.14 about projective arrangements. The advantage of the proof below is that it uses the Homotopy Lemma in its simplest form and allows a uniform treatment of all the special cases above. It is clear that this method may be useful for other applications, say for other group actions, since the argument no longer requires the arrangement to be shifted to a more special position by a dilatation $e_{i} \mapsto \varepsilon^{i} e_{i}, i=1, \ldots, n$, for some $\varepsilon>0$.

Our objective is to show that the homotopy type of the link has a purely combinatorial description in terms of the poset $P$ and the associated rank function $d: P \rightarrow \mathbb{N}_{0}$. The link of a (spherical, affine, projective, lens, Grassmann etc.) arrangement is the union of all spaces in the arrangement. It follows from the Projection Lemma that the link has the same homotopy type as the homotopy colimit of the corresponding diagram.

We uniformly construct a combinatorially defined diagram which serves for comparison with the original one. Choose a flag $F=\left\{F_{i}\right\}_{i=0}^{n},\{\boldsymbol{0}\}=F_{0} \subset F_{1} \subset \cdots \subset F_{n}=\mathbb{K}^{n}$. Let $V \mapsto T(V)$ be one of the functors described above. Then $T[F]=T[F](A)$, the flag diagram associated with $T(A)$, is defined by $T[F]_{p}:=T\left(F_{d(p)}\right)$ where the morphisms $T[F]_{p} \rightarrow T[F]_{q}$ are the obvious inclusion maps. Every two flag diagrams $T[F]$ and $T\left[F^{\prime}\right]$ are naturally isomorphic, thus the isomorphism type of $T[F]$ depends only on $P$ and $d$.

We want to compare our diagram $T(A)$ with the combinatorially defined diagram $T[F](A)$. There does not seem to exist a natural map of diagrams between $T(A)$ and

10 Journal für Mathematik. Band 509 
$T[F](A)$ because e.g. the projection map is not natural. This difficulty was overcome in [49], in the case of projective diagrams, by shifting the diagram $T[F](A)$ to a more special position and by applying a more general version of the Homotopy Lemma by Vogt [44] that allows noncommutative diagrams if they commute up to coherent homotopies. The proof of Theorem 5.8 shows that in practical problems a very natural idea to use is the comparison with the third, so called "ample space" diagram that contains both $T(A)$ and $T[F](A)$ as subdiagrams. We need a lemma which explains what is meant by "ample" in all the interesting cases above.

Lemma 5.7. Let $T: \operatorname{Vect}\left(\mathbb{K}^{n}\right) \rightarrow$ Top, $V \mapsto T(V)$, be one of the functors defined above which to every vector space $V \subset \mathbb{K}^{n}$ associates the corresponding projective space $P(V)$, Grassmann manifold $G_{k}(V)$, the lens space $L_{m}(V)\left(\right.$ for $\left.V \subset \mathbb{C}^{n}\right)$, or the unit sphere $S(V)$. Then in each of these cases there exists a functor $R_{T}: \operatorname{Vect}\left(\mathbb{K}^{n}\right) \rightarrow$ Top which associates with each subspace $V \subset \mathbb{K}^{n}$ an "ample" subspace $R_{T}(V) \subset T\left(\mathbb{K}^{n}\right)$ and to each inclusion $V \rightarrow W$ an inclusion of spaces $R_{T}(V) \rightarrow R_{T}(W)$ so that the following condition is satisfied.

Let $V \subset \mathbb{K}^{n}, \operatorname{dim}(V)=k$. Then for any $W$ of dimension $k$ with $\operatorname{dim}\left(W \cap V^{\perp}\right)=0$, there is an inclusion $T(W) \subset R_{T}(V)$ so that the inclusion map

$$
i_{W}: T(W) \rightarrow R_{T}(V)
$$

is a homotopy equivalence (actually, an inverse to a deformation retraction).

Proof. The space $R_{T}(V)$ is defined in a very similar way for all the examples above. For example in the case of the functor $V \mapsto S(V)$, we put $R_{T}(V):=S\left(\mathbb{K}^{n}\right) \backslash S\left(V^{\perp}\right)$. The construction in the case of a projective functor $V \mapsto P(V)$ is analogous, one removes the projective space $P\left(V^{\perp}\right)$ from $P\left(\mathbb{K}^{n}\right)$. The inclusion map $i_{V}: P(V) \rightarrow P\left(\mathbb{K}^{n}\right) \backslash P\left(V^{\perp}\right)$ is a homotopy equivalence since $P\left(\mathbb{K}^{n}\right) \backslash P\left(V^{\perp}\right)$ is the total space of a vector space bundle (a tubular neighborhood) over $P(V)$. Obviously $P(W) \subset P\left(\mathbb{K}^{n}\right) \backslash P\left(V^{\perp}\right)$ for any $W$ with the property $W \cap V^{\perp}=\{0\}$. Finally, $i_{W}: P(W) \rightarrow R_{T}(V)$ is a homotopy equivalence since there exists a linear map $O_{V, W}: \mathbb{K}^{n} \rightarrow \mathbb{K}^{n}$ which maps $V$ to $W$ and leaves $V^{\perp}$ invariant. Something similar is done in the case of Grassmannians. Here, $R_{G_{k}}(V)$ is defined by

$$
R_{G_{k}}(V):=\left\{L \in G_{k}\left(\mathbb{K}^{n}\right): \operatorname{dim}\left(L \cap V^{\perp}\right)=0\right\}
$$

In this case $R_{G_{k}}(V)$ is also the total space of a vector bundle over $G_{k}(V)$ which can be seen as follows. If $M_{k}(V)$ is the Stiefel manifold of all 1-1 linear maps (matrices) $D: \mathbb{K}^{k} \rightarrow V$ then $G_{k}(V)=M_{k}(V) / G(k)$ where $G(k)=\mathrm{GL}(k, K)$ is the appropriate group of linear automorphisms. Let $p: M_{k}\left(\mathbb{K}^{n}\right) \rightarrow G_{k}\left(\mathbb{K}^{n}\right)$ be the projection map. Note that

$$
p^{-1}\left(R_{G_{k}}(V)\right)=M_{k}(V) \times L\left(\mathbb{K}^{k}, V^{\perp}\right),
$$

where $L\left(\mathbb{K}^{k}, V^{\perp}\right)$ is the space of all linear maps, and that the group $G(k)$ acts diagonally on the product $M_{k}(V) \times L\left(\mathbb{K}^{k}, V^{\perp}\right)$. Now it is enough to recall that if $X$ and $Y$ are two $G$ spaces and if the action on $Y$ is free, then the orbit space $(X \times Y) / G$ of the diagonal action is represented as a fiber bundle

$$
X \rightarrow(X \times Y) / G \rightarrow Y / G
$$


which in our case means that $R_{G_{k}}(V)$ is fibered over $G_{k}(V)$ with the fiber $L\left(\mathbb{K}^{k}, V^{\perp}\right)$. Hence, $i_{V}$ is a homotopy equivalence. It is shown that $i_{W}$ is also a homotopy equivalence analogously to the case of projective diagrams.

Finally, in the case of the "lens space" functor, let $R_{L_{m}}(V):=L_{m}\left(\mathbb{C}^{n}\right) \backslash L_{m}\left(V^{\perp}\right)$. The proof that $R_{L_{m}}(V)$ is "ample" in the sense above is analogous and can rely on the fact that the sphere $S\left(\mathbb{C}^{n}\right)$ is a join of spheres $S(V)$ and $S\left(V^{\perp}\right)$, and that the action of the cyclic group $\mathbb{Z}_{m}$ respects this decomposition.

Theorem 5.8 (Homotopy types of arrangements). Let $A=\left\{A_{p}\right\}_{p \in P}$ be a linear subspace arrangement of $\mathbb{K}^{n}$, where $\mathbb{K}$ is one of the (skew) fields $\mathbb{R}, \mathbb{C}$ or $\mathbb{H}$. Let $P$ be the intersection poset of $A$, with the dimension function $d: P \rightarrow \mathbb{N}_{0}, d(p)=\operatorname{dim}\left(A_{p}\right)$, defined above, and set $P_{[k]}:=\{p \in P: d(p) \geqq k\}$ for $k \geqq 0$.

Let $T:$ Vect $\rightarrow$ Top, $V \mapsto T(V)$, be the projective, sphere, Grassmann or the lens space functor defined above and let $T(A)$ be the corresponding arrangement of subspaces of $T\left(\mathbb{K}^{n}\right)$. Then there is a homotopy equivalence

$$
\bigcup_{p \in P} T\left(A_{p}\right) \simeq \bigcup_{k=0}^{n} T\left(\mathbb{K}^{k}\right) \times \Delta\left(P_{[k]}\right)
$$

Proof. We start by choosing the flag $F, F_{i}=\operatorname{span}\left\{e_{k}\right\}_{k=1}^{i}$, in sufficiently general position with respect to the arrangement $A$. This requirement means that for any $F_{i}$ and $A_{p} \in A$, if $\operatorname{dim}\left(F_{i}\right)=\operatorname{dim}\left(A_{p}\right)$ the $A_{p} \cap F_{i}^{\perp}=\{0\}$. Let us define the associated "ample" space diagram $\mathscr{R}=\mathscr{R}(A)$ by $\mathscr{R}=\left\{R_{p}\right\}_{p \in P}, R_{p}:=R_{T}\left(A_{p}\right)=R_{T}\left(F_{d(p)}\right)$, where $V \mapsto R_{T}(V)$ is the "ample" space functor associated with $T$ described in Lemma 5.7. Hence, there exist two naturally defined maps of diagrams $\alpha$ and $\beta$,

$$
\alpha: T(A) \rightarrow \mathscr{R}(A) \leftarrow T[F](A): \beta
$$

induced by the inclusion maps $\alpha_{p}: T\left(A_{p}\right) \rightarrow R_{p}$ and $\beta_{p}: T[F]_{p} \rightarrow R_{p}$. By Lemma 5.7 these inclusion maps are homotopy equivalences. From here and the Homotopy Lemma it follows that hocolim $T(A) \simeq \operatorname{hocolim} \mathscr{R}$ and hocolim $T[F](A) \simeq$ hocolim $\mathscr{R}$, which implies

$$
\operatorname{hocolim} T(A) \simeq \operatorname{hocolim} T[F](A)
$$

Finally, we notice that the embeddings $T\left(F_{k}\right) \hookrightarrow T\left(\mathbb{K}^{n}\right)$ induce a diagram map

$$
\Xi: T[F](A) \hookrightarrow \mathscr{F},
$$

where $\mathscr{F}$ denotes the "constant" $P$-diagram which has $F_{p}=T\left(\mathbb{K}^{n}\right)$ for all $p \in P$, and identity maps $f_{p p^{\prime}}$ for $p \geqq p^{\prime}$. From the Embedding Lemma 4.10 we get that $\hat{\Xi}$ is an embedding

$$
\hat{\Xi}: \operatorname{hocolim} T[F](A) \hookrightarrow \operatorname{hocolim} \mathscr{F} \cong T\left(\mathbb{K}^{n}\right) \times \Delta(P),
$$

and we easily identify the image of $\hat{\Xi}$ with the space $\bigcup_{k=0}^{n} T\left(\mathbb{K}^{k}\right) \times \Delta\left(P_{[k]}\right)$. 
Note that for the homotopy formula for Grassmann arrangements in Theorem 6.4 only the truncated poset $P_{[k]}=\{p \in P: d(p) \geqq k\}$ is relevant: This corresponds to the fact that spaces with $d(p)<k$ do not have $k$-dimensional subspaces, so $A$ and

$$
A_{[k]}=\left\{A_{p} \in A: d(p) \geqq k\right\}
$$

have the same associated $k$-Grassmann arrangement.

The following proposition shows that there is a general decomposition formula for the homology of flag diagrams and, a posteriori, of the $T$-links of $\mathbb{K}$-arrangements for $\mathbb{K}=\mathbb{R}, \mathbb{C}$ or $\mathbb{H}$.

Proposition 5.9 (Homology of flag diagrams). Let $A$ be an arrangement of linear subspaces in $\mathbb{K}^{n}$ and let $T$ be one of the functors described above. Let $T[F](A)$ be the combinatorially defined flag diagram associated to the arrangement $A$ and the functor $T$. We set $s=\min \left\{d\left(A_{p}\right)\right\}_{p \in P}$ and $t=\max \left\{d\left(A_{p}\right)\right\}_{p \in P}$. Assume that for the coefficient ring $R$ and for all $s \leqq k \leqq m \leqq t$

- the exact sequence of the pair $\left(T\left(\mathbb{K}^{m}\right), T\left(\mathbb{K}^{k}\right)\right)$ splits in homology and

- the homology groups $H_{*}\left(T\left(\mathbb{K}^{m}\right) ; R\right)$ are free $R$-modules.

Then,

$$
\begin{gathered}
H_{*}(\operatorname{hocolim} T[F](A) ; R) \\
\cong\left(H_{*}\left(\Delta\left(P_{[s]}\right) ; R\right) \otimes H_{*}\left(T\left(\mathbb{K}^{s}\right) ; R\right)\right) \oplus \bigoplus_{k=s+1}^{t}\left(H_{*}\left(\Delta\left(P_{[k]}\right) ; R\right) \otimes H_{*}\left(T\left(\mathbb{K}^{k}\right), T\left(\mathbb{K}^{k-1}\right) ; R\right)\right) .
\end{gathered}
$$

Proof. For this proof we fix the coefficient ring $R$ used for homology computations and we write $H_{*}(\cdot)$ for $H_{*}(\cdot ; R)$. For an arbitrary poset $Q$ and a natural number $k$ we introduce "constant" diagrams $\mathscr{M}_{k, Q}$ over $Q$, defined by $\mathscr{M}_{k, Q}: Q \rightarrow T o p, \mathscr{M}_{k, Q}(q):=T\left(\mathbb{K}^{k}\right)$. If it is clear from the context which poset $Q$ is used, we write $\mathscr{M}_{k}$ for the diagram $\mathscr{M}_{k, Q}$. Note that our diagram $T[F](A)$ can be squeezed in between two constant diagrams $\mathscr{M}_{s, P}$ and $\mathscr{M}_{t, P}$. Clearly, hocolim $\mathscr{M}_{k, Q} \cong \Delta(Q) \times T\left(\mathbb{K}^{k}\right)$. The Künneth theorem and the freeness of $R$-modules $H_{*}\left(T\left(\mathbb{K}^{k}\right)\right)$ imply

$$
H_{*}\left(\operatorname{hocolim} \mathscr{M}_{s, Q}\right) \cong H_{*}(\Delta(Q)) \otimes H_{*}\left(T\left(\mathbb{K}^{s}\right)\right) .
$$

From this observation and from the fact that the map $H_{*}\left(T\left(\mathbb{K}^{k}\right)\right) \rightarrow H_{*}\left(T\left(\mathbb{K}^{m}\right)\right)$ is a monomorphism for $k \leqq m$, we conclude that the map hocolim $\mathscr{M}_{k} \rightarrow$ hocolim $\mathscr{M}_{m}$, for $k \leqq m$, induces a monomorphism of homology groups. Then the composition of homomorphisms

$$
H_{*}\left(\operatorname{hocolim} \mathscr{M}_{s}\right) \rightarrow H_{*}(\operatorname{hocolim} T[F](A)) \rightarrow H_{*}\left(\operatorname{hocolim} \mathscr{M}_{t}\right)
$$

is a monomorphism too. Thus the first of them is also a monomorphism. It follows that the long exact sequence of the pair (hocolim $T[F](A)$, hocolim $\left.\mathscr{M}_{s}\right)$ splits and

$$
H_{*}(\operatorname{hocolim} T[F](A)) \cong H_{*}\left(\operatorname{hocolim} \mathscr{M}_{s}\right) \oplus H_{*}\left(\operatorname{hocolim} T[F](A), \operatorname{hocolim} \mathscr{M}_{s}\right)
$$


Informally speaking, we peel from the homotopy colimit of the diagram $T[F](A)$ the part of its homology coming from the constant subdiagram $\mathscr{M}_{s}$. Let $\mathscr{E}_{[i]}$ be the restriction of the diagram $T[F](A)$ on the poset $P_{[s+i]}, i \geqq 0$. By excision, recalling the definition of the homotopy colimit hocolim $T[F](A)$, we have

$$
H_{*}\left(\operatorname{hocolim} T[F](A), \operatorname{hocolim} \mathscr{M}_{s}\right) \cong H_{*}\left(\operatorname{hocolim} \mathscr{E}_{[1]}, \operatorname{hocolim} \mathscr{M}_{s, P_{[s+1]}}\right)
$$

By induction on $i$ we may assume that for some $i \geqq 0$

$$
\begin{aligned}
H_{*}(\text { hocolim } T[F](A)) \cong & \left(H_{*}\left(\Delta\left(P_{[s]}\right)\right) \otimes H_{*}\left(T\left(\mathbb{K}^{s}\right)\right)\right) \\
& \oplus \bigoplus_{\substack{k+s+1 \\
s+i}}\left(H_{*}\left(\Delta\left(P_{[k]}\right)\right) \otimes H_{*}\left(T\left(\mathbb{K}^{k}\right), T\left(\mathbb{K}^{k-1}\right)\right)\right) \\
& \oplus H_{*}\left(\text { hocolim } \mathscr{E}_{[i+1]}, \text { hocolim } \mathscr{M}_{s+i, P_{[s+i+1]}}\right) .
\end{aligned}
$$

The long exact sequence of the triple

$$
\left(\operatorname{hocolim} \mathscr{E}_{[i+1]}, \text { hocolim } \mathscr{M}_{s+i+1, P_{[s+i+1]}}, \operatorname{hocolim} \mathscr{M}_{s+i, P_{[s+i+1]}}\right)
$$

splits by the same argument as above. This means that we can peel from the homology $H_{*}\left(\right.$ hocolim $\mathscr{E}_{[i+1]}$, hocolim $\left.\mathscr{M}_{s+i, P_{[s+i+1]}}\right)$ the part isomorphic to

$$
\begin{aligned}
& H_{*}\left(\text { hocolim } \mathscr{M}_{s+i+1, P_{[s+i+1]}}, \text { hocolim } \mathscr{M}_{s+i, P_{[s+i+1]}}\right) \\
& \cong H_{*}\left(\Delta\left(P_{[s+i+1]}\right)\right) \otimes H_{*}\left(T\left(\mathbb{K}^{s+i+1}\right), T\left(\mathbb{K}^{s+i}\right)\right)
\end{aligned}
$$

The part that remains is isomorphic to the group $H_{*}\left(\right.$ hocolim $\left.\mathscr{E}_{[i+1]}, \mathscr{M}_{s+i+1, P_{[s+i+1]}}\right)$. The last group is by excision isomorphic to $H_{*}$ (hocolim $\mathscr{E}_{[i+2]}$, hocolim $\mathscr{M}_{s+i+1, P_{[s+i+2]}}$ ). So the Künneth formula, the process described above and induction on $i$ lead to the desired formula.

If the arrangement $A$ is essential (i.e., if $s=0$ and $\left.t=\max \left\{d\left(A_{p}\right)\right\}_{p \in P}=n\right)$, then both $\mathbb{K}^{-1}$ and $T\left(\mathbb{K}^{-1}\right)$ are interpreted as empty spaces and the formula given in Proposition 5.9 can be rewritten as follows.

Corollary 5.10. Let $A$ be an essential arrangement satisfying the assumptions of Proposition 5.9. Then

$$
H_{*}(\operatorname{hocolim} T[F](A) ; R) \cong \bigoplus_{k=0}^{n} H_{*}\left(\Delta\left(P_{[k]}\right) ; R\right) \otimes H_{*}\left(T\left(\mathbb{K}^{k}\right), T\left(\mathbb{K}^{k-1}\right) ; R\right)
$$

For example if $T=P$ is the functor which associates the complex projective space $P(V)$ to every complex linear space $V$, then the formula given in Corollary 5.10 has the following form

$$
H_{r}(\operatorname{hocolim} T[F](A) ; R) \cong \bigoplus_{k=0}^{n} H_{r-2 k}\left(\Delta\left(P_{[k]}\right) ; R\right)
$$


where $r \geqq 0$ and $H_{-k}(X ; R):=0$ for all $k>0$. This formula together with its counterpart for the real projective arrangements was formulated and proved in [49]. Unfortunately, as it was kindly pointed to us by Anders Björner and Karanbir Sarkaria, the formulation there suffers from some misprints.

The following example, which we also owe to A. Björner and K. Sarkaria, shows how a formula of the type above arises in connection with the Stanley-Reisner ring of a simplicial complex $\Delta$.

Corollary 5.11. Let $\Delta$ be a simplicial complex on the vertex set $\{1, \ldots, n\}$. Let $A_{\Delta}$ be the arrangement of complex linear subspaces in $\mathbb{C}^{n}$ defined by $A_{\Delta}=\left\{A_{\sigma}\right\}_{\sigma \in \Delta}$, where $A_{\sigma}:=\operatorname{span}_{\mathbb{C}}\left\{e_{j}\right\}_{j \in \sigma}, e_{i}$ the ith unit coordinate vector in $\mathbb{C}^{n}$. Let $P\left(A_{\Delta}\right)=\left\{P\left(A_{\sigma}\right)\right\}_{\sigma \in \Delta}$ be the associated projective arrangement. Then the homology of the union $\bigcup_{\sigma \in \Delta} P\left(A_{\sigma}\right)$ of the arrange-
ment $P(A)$ is given by

$$
H_{r}\left(\bigcup_{\sigma \in \Delta} P\left(A_{\sigma}\right) ; \mathbb{Z}\right) \cong \bigoplus_{k=0}^{n} H_{r-2 k}\left(\Delta\left(\Delta_{[k]}\right) ; \mathbb{Z}\right),
$$

where $\Delta_{[k]}:=\{\sigma \in \Delta: \operatorname{dim}(\sigma) \geqq k\}$ is a subposet of $(\Delta, \subset)$ and $\Delta\left(\Delta_{[k]}\right)$ its order complex.

Note that the union of the arrangement $P\left(A_{\Delta}\right)$ is a projective variety whose homogeneous coordinate ring is the Stanley-Reisner ring of $\Delta$.

Note that Proposition 5.9 deals with the case when $H_{*}\left(T\left(\mathbb{K}^{i}\right)\right) \rightarrow H_{*}\left(T\left(\mathbb{K}^{i+1}\right)\right)$ is injective in contrast to the Goresky-MacPherson formula (Corollary 5.6). We already mentioned that the Goresky-MacPherson formula for the cohomology of the complement of an arrangement $A$ of (affine) subspaces can be proved by Alexander duality from the homology of an associated arrangement $S(A)$ of spheres. In the case when $T=S$ the map $\tilde{H}_{*}\left(T\left(\mathbb{K}^{i}\right)\right) \rightarrow \tilde{H}_{*}\left(T\left(\mathbb{K}^{i+1}\right)\right)$ is trivial. Although it does not completely fit in the setting of this section one may regard toric varieties - seen from the point of view of Section 5.3 as an interesting third case, when the map in homology induced by the diagram maps are surjective.

5.5. Subgroup complexes. The order complex of the poset

$$
S_{p}(G)=\left\{P \leqq G:|P|=p^{i} \neq 1\right\}
$$

of non-trivial $p$-subgroups of a finite group $G$ has received considerable interest over that past few years (see for example [1]). It was already observed by Quillen [34] that $S_{p}(G)$ is homotopy equivalent to the poset $A_{p}(G)$ of non-trivial elementary abelian $p$-subgroups of $G$. In [31] the authors consider the covering of $\Delta\left(A_{p}(G)\right)$ by the subcomplexes $\Delta\left(A_{p}(N A)\right)$ for a fixed solvable normal $p^{\prime}$-subgroup $N$ and maximal elementary abelian $p$-subgroups $A$ of $G$. Then they use the following facts:

(a) Intersections of the spaces of type $\Delta\left(A_{p}(N A)\right)$ are again of the type $\Delta\left(A_{p}(N D)\right)$ for some elementary abelian $p$-subgroup $D$ of $G$. 
(b) For a solvable normal $p^{\prime}$-group $N$ and an elementary abelian $p$-subgroup $A$ the complex $\Delta\left(A_{p}(N A)\right)$ is homotopy equivalent to a wedge of spheres of dimension $\operatorname{rank}(D)-1$.

Observation (a) follows by basic group theoretical argumentation. Assertion (b) is much less obvious. It was established by Quillen [34], Theorem 11.2, but also follows by applications of the homotopy colimit methods (see [31], Theorem (A)). Using facts (a) and (b), the Projection, the Homotopy and the Wedge Lemma the following wedge decomposition of $\Delta\left(A_{p}(G)\right)$ for finite solvable groups $G$ with non-trivial normal $p^{\prime}$-group is proved in [31].

Theorem 5.12 (Pulkus and Welker [31], Theorem (B)). Let $G$ be a finite group and let $p$ be a prime. Let $N$ be a solvable normal $p^{\prime}$-subgroup. Let $C N / N$ be the intersection of all maximal elementary abelian p-subgroups of $G / N$. For $A N / N \in A_{p}(G / N)$ let $c_{A N / N}$ be an arbitrary but fixed point in $\Delta\left(A_{p}(G / N)_{>A N / N}\right)$. Then $\Delta\left(S_{p}(G)\right)$ is homotopy equivalent to

$$
\underset{A N / N \in A_{p}(G / N)>C N / N \cup\{C N / N\}}{\bigvee} \Delta\left(A_{p}(N A)\right) * \Delta\left(A_{p}(G / N)_{>A N / N}\right)
$$

where the wedge is formed by identifying, for $A N / N>N / N$,

$$
\text { the point } c_{A N / N} \in \Delta\left(A_{p}(G / N)_{>A N / N}\right) * A_{p}(N A)
$$

with the point $A N / N \in \Delta\left(A_{p}(G / N)\right) * \Delta\left(A_{p}(N 1)\right)$.

In particular, if $A$ is a maximal elementary abelian group of rank $r$ in $G$, then $\Delta\left(A_{p}(N A)\right) * \Delta\left(A_{p}(G / N)_{>A N / N}\right)$ is homotopyc to a wedge of $(r-1)$-spheres.

Acknowledgments. Thanks to Eva-Maria Feichtner for helpful comments on the manuscript. We are grateful to Emmanuel Dror Farjoun and Rainer Vogt for some corrections and very valuable remarks and references. The referee's remarks, corrections and additions (see in particular Section 3.3) were extremely helpful, and led to a substantial improvement of the paper and its exposition.

We all thank the Konrad-Zuse-Zentrum in Berlin for its hospitality.

\section{References}

[1] M. Aschbacher and S. Smith, On Quillen's conjecture for the p-subgroups complex, Ann. Math. (2) 137 (1993), 473-529.

[2] E. Babson, A Combinatorial Flag Space, PhD thesis, MIT, 1993.

[3] C. Balteanu, Z. Fiedorowicz, R. Schwänzl, R. Vogt, Iterated monoidal categories II, preprint 1998.

[4] A. Björner, Topological methods, in: R. Graham, M. Grötschel and L. Lovász, ed., Handbook of Combinatorics, North-Holland, Amsterdam (1995), 1819-1872.

[5] A. Björner, A generalized homotopy complementation formula, Discr. Math. 193 (1998), 85-91.

[6] A. Björner and J.W. Walker, A homotopy complementation formula for partially ordered sets, Europ. J. Comb. 4 (1983), 11-19.

[7] M. Bousfield and D.M. Kan, Homotopy Limits, Completions and Localizations, Springer Lect. Notes Math. 304, Berlin-Heidelberg-New York 1972.

[8] R. Brown, Elements of Modern Topology, McGraw-Hill, London 1968. 
[9] V. I. Danilov, The geometry of toric varieties, Russ. Math. Surv. 33(2) (1978), 97-151.

[10] A. Dold and R. Thom, Quasifaserungen und unendliche symmetrische Produkte, Ann. Math. 67(2) (1958), $239-281$.

[11] W.G. Dwyer and D.M. Kan, Function complexes for diagrams of simplicial sets, Proc. Kon. Ned. Akad. van Wetensch. A 86 (the same as) Ind. Math. 45 (1983), 139-147.

[12] W.G. Dwyer and D.M. Kan, A classification theorem for diagrams of simplicial sets, Topology 23(2) (1984), $139-155$

[13] G. Ewald, Combinatorial Convexity and Algebraic Geometry, Springer Grad. Texts Math. 168, BerlinHeidelberg-New York 1996

[14] E. Dror Farjoun, Homotopy and homology of diagrams of spaces, in: Proc. Workshop "Algebraic Topology" (Seattle/Wash. 1985), Springer Lecture Notes Math. 1286, (1987), 93-124.

[15] E. Dror Farjoun, Cellular Spaces, Null Spaces and Homotopy Localization, Springer Lect. Notes Math. 1622, Berlin-Heidelberg-New York 1991.

[16] E. Dror Farjoun and A. Zabrodsky, Homotopy equivalence between diagrams of spaces, J. Pure Appl. Algebra 41 (1986), 169-182.

[17] S. Fischli and D. Yavin, Which 4-manifolds are toric varieties? Math. Z. 215 (1994), 179-185.

[18] W. Fulton, Introduction to Toric Varieties, Ann. Math. Stud. 131, Princeton University Press, 1993.

[19] P. Gabriel and M. Zisman, Calculus of Fractions and Homotopy Theory, Springer Erg. Math. Grenzgeb. 35, Berlin-New York 1967.

[20] M. Goresky and R. MacPherson, Stratified Morse Theory, Springer Erg. Math. Grenzgeb. 14, BerlinHeidelberg-New York 1988

[21] J. Hollender and R.M.Vogt, Modules of topological spaces, applications to homotopy limits and $E_{x}$ structures, Arch. Math. (Basel) 59 (1992), 115-129.

[22] J.F. Kraus, Mayer-Vietoris-Prinzipien for Cofaserungen, Math. Ann. 231 (1977), 47-53.

[23] J. Lillig, A union theorem for cofibrations, Arch. Math. (Basel) 24 (1973), 410-415.

[24] A.T. Lundell and S. Weingram, The Topology of $C W$ Complexes, Van Nostrand Reinhold, New York 1966

[25] J.P. May, Simplicial Objects in Algebraic Topology, Van Nostrand Math. Stud. 11, Princeton NJ 1967.

[26] J. P. May, The Geometry of Iterated Loop Spaces, Springer Lect. Notes Math. 271, Berlin-Heidelberg-New York 1972.

[27] J.P. May, $E_{\infty}$-spaces, group completions and permutative categories, in: New Developments in Topology (G. Segal, ed.), London Math. Soc. Lect. Note Ser. 11 (1974), 61-93.

[28] M. Mc Connell, Rational homology of toric varieties, Proc. Amer. Math. Soc. 105(4) (1989), 986-991.

[29] J. Milnor, The geometric realization of a semi-simplicial complex, Ann. Math. (2) 65 (1957), 357-362.

[30] J. Milnor, On spaces having the homotopy type of a $C W$-complex, Trans. Amer. Math. Soc. 90 (1959), $272-280$.

[31] J. Pulkus and $V$. Welker, On the homotopy type of the p-subgroup complex for finite solvable groups, preprint 1995.

[32] D. Puppe, Homotopiemengen und induzierte Abbildungen, I, Math. Z. 69 (1958), 299-344.

[33] D. Quillen, Higher Algebraic $K$-Theory: I, Springer Lect. Notes Math. 341, Berlin-Heidelberg-New York 1973.

[34] D. Quillen, Homotopy properties of the poset of nontrivial $p$-subgroups of a group, Adv. Math. 28 (1978), 101-128.

[35] K.S. Sarkaria, A generalized Kneser conjecture, J. Comb. Th. (B) 49 (1990), 236-240.

[36] C. Schaper, Suspensions of affine arrangements, Math. Ann. 309 (1997), 463-473.

[37] G. B. Segal, Classifying spaces and spectral sequences, Publ. Math. I.H.E.S. 34 (1968), 105-112.

[38] G. B. Segal, Categories and cohomology theories, Publ. Math. I.H.E.S. 13 (1974), 293-312.

[39] R.P. Stanley, Enumerative Combinatorics, I, Wadsworth and Brooks/Cole, Monterey, CA, 1986.

[40] B. Sturmfels and G.M. Ziegler, Extension spaces of oriented matroids, Disc. Comp. Geom. 10 (1993), $23-45$.

[41] S. Sundaram and $V$. Welker, Group actions on arrangements of linear subspaces and applications to configuration spaces, Trans. Amer. Math. Soc. 239 (1997), 1389-1420.

[42] T. tom Dieck, Partitions of unity in homotopy theory, Comp. Math. 23 (1973), 159-161.

[43] V.A.Vassiliev, Complements of Discriminants of Smooth Maps: Topology and Applications, Transl. Math. Monogr. 98, Amer. Math. Soc., Providence, RI, revised edition 1994.

[44] R.M.Vogt, Homotopy limits and colimits, Math. Z. 134 (1973), 11-52.

[45] J.W.Walker, Homotopy type and Euler characteristic of partially ordered sets, Europ. J. Combin. 2 (1981), $373-384$

[46] J.W.Walker, Canonical homeomorphisms of posets, Europ. J. Combin. 9 (1988), 97-107. 
[47] G.W.Whitehead, Elements of Homotopy Theory, Springer Grad. Texts Math. 61, Berlin-Heidelberg-New York 1978.

[48] D. Yavin, The Intersection Homology with Twisted Coefficients of Toric Varieties, PhD thesis, MIT, 1990.

[49] G.M. Ziegler and R.T. Živaljević, Homotopy types of subspace arrangements via diagrams of spaces, Math. Ann. 295 (1993), 527-548.

[50] R.T. Živaljević, Topological methods, in: CRC Handbook on Discrete and Computational Geometry (J. E. Goodman, J. O'Rourke, eds.), CRC Press, Boca Raton (1997), 209-224.

[51] R.T. Živaljevic, Combinatorics of topological posets: Homotopy complementation formulas, Adv. Appl. Math. 21 (1998), 547-574.

Fachbereich Mathematik, MA 7-1, Technische Universität Berlin, D-10623 Berlin e-mail: welker@math.tu-berlin.de e-mail: ziegler@math.tu-berlin.de

Mathematics Institute, Knez Mihailova 35/1, P. F. 367, YU-11001 Belgrade e-mail: rade@turing.mi.sanu.ac.yu

Eingegangen 5. April 1995, in revidierter Fassung 1. Juli 1998 
Bereitgestellt von | Technische Universität Berlin Angemeldet Heruntergeladen am | 09.11.18 17:08 